\title{
Mathematics of Photoacoustic and Thermoacoustic Tomography
}

\author{
P. Kuchment and L. Kunyansky \\ Contact information: \\ Peter Kuchment \\ Mathematics Department \\ Texas A \& M University \\ College Station, TX USA 77843-3368 \\ phone: (979) 862-3257, FAX: (979) 862-4190 \\ e-mail: kuchment@math.tamu.edu \\ Web: http://www.math.tamu.edu/ ${ }^{\sim}$ kuchment \\ Leonid Kunyansky \\ Department of Mathematics \\ University of Arizona, AZ USA 85721 \\ phone: (520)621-4509, FAX: (520)621-8322 \\ e-mail: leonk@math.arizona.edu
}




\begin{abstract}
This is the manuscript of the chapter for a planned Handbook of Mathematical Methods in Imaging that surveys the mathematical models, problems, and algorithms of the Thermoacoustic (TAT) and Photoacoustic (PAT) Tomography. TAT and PAT represent probably the most developed of the several novel "hybrid" methods of medical imaging. These new modalities combine different physical types of waves (electromagnetic and acoustic in case of TAT and PAT) in such a way that the resolution and contrast of the resulting method are much higher than those achievable using only acoustic or electromagnetic measurements.
\end{abstract}

\title{
Contents
}

1 Introduction $\quad 3$

2 Mathematical models of TAT 4

2.1 Point detectors and the wave equation model . . . . . . . . . . . . . . . . 4

2.2 Acoustically homogeneous media and spherical means . . . . . . . . . . . . 5

2.3 Main mathematical problems arising in TAT . . . . . . . . . . . . . 6

2.4 Variations on the theme: planar, linear, and circular integrating detectors . . 7

3 Mathematical analysis of the problem $\quad 9$

3.1 Uniqueness of reconstruction . . . . . . . . . . . . . . . . . . . . . . 9

3.1.1 Acoustically homogeneous media . . . . . . . . . . . . . . . 10

3.1.2 Acoustically inhomogeneous media . . . . . . . . . . . . . . . . 14

3.2 Stability . . . . . . . . . . . . . . . . . . . 16

3.3 Incomplete data . . . . . . . . . . . . . . . . . . . . . . . . . . . . . . . . . . . . . . . . . . .

3.3.1 Uniqueness of reconstruction . . . . . . . . . . . . . . . 17

3.3.2 "Visible" ("audible") singularities . . . . . . . . . . . . . . . . . . . 19

3.3.3 Stability of reconstruction for incomplete data problems . . . . . . . 21

3.4 Discussion of the visibility condition . . . . . . . . . . . . . . . . . 21

3.5 Range conditions . . . . . . . . . . . . . . . . . . . . 22

3.5.1 The range of the spherical mean operator $\mathcal{M} . \ldots . . . . . .24$

3.5.2 The range of the forward operator $\mathcal{W}$. . . . . . . . . . . . . . 25

3.6 Speed of sound reconstruction . . . . . . . . . . . . . . . . . . 26

4 Reconstruction formulas and procedures $\quad 27$

4.1 Full data (closed acquisition surfaces) . . . . . . . . . . . . . . . . 27

4.1.1 Constant speed of sound . . . . . . . . . . . . . . 27

4.1.2 Variable speed of sound . . . . . . . . . . . . . . . . . . . . . . . . . . . . . . . . 35

4.2 Partial (incomplete) data . . . . . . . . . . . . . . . . . . . . . . . . . . . . . . . . . 37

4.2.1 Constant speed of sound . . . . . . . . . . . . . . . . 38

4.2.2 Variable speed of sound . . . . . . . . . . . . . 40

$5 \quad$ Final remarks and open problems $\quad 41$ 


\section{Introduction}

We provide here just a very brief description of the TAT/PAT procedure, since the relevant physics and biology details can be found in another chapter [132] in this volume, as well as in the surveys and books $[133,134,137]$. In TAT (PAT), a short pulse of radio-frequency EM wave (correspondingly, laser beam) irradiates a biological object (e.g., in the most common application, human breast), thus causing small levels of heating. The resulting thermoelastic expansion generates a pressure wave that starts propagating through the object. The absorbed EM energy and the initial pressure it creates are much higher in the cancerous cells than in healthy tissues (see the discussion of this effect in $[132-134,137]$ ). Thus, if one could reconstruct the initial pressure $f(x)$, the resulting TAT tomogram would contain highly useful diagnostic information. The data for such a reconstruction are obtained by measuring time-dependent pressure $p(x, t)$ using acoustic transducers located on a surface $S$ (we will call it the observation or acquisition surface) completely or partially surrounding the body (see Fig. 1). Thus, although the initial irradiation is electro-magnetic, the actual recon-

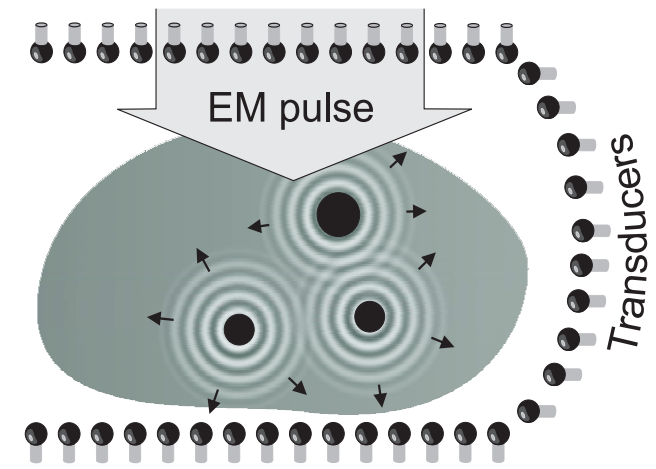

Transducers

Figure 1: TAT/PAT procedure with a partially surrounding acquisition surface.

struction is based on acoustic measurements. As a result, the high contrast is produced due to a much higher absorption of EM energy by cancerous cells (ultrasound alone would not produce good contrast in this case), while the good (sub-millimeter) resolution is achieved by using ultrasound measurements (the radio frequency EM waves are too long for highresolution imaging). Thus, TAT, by using two types of waves, combines their advantages, while eliminating their individual deficiencies.

The physical principle upon which TAT/PAT is based was discovered by Alexander Graham Bell in 1880 [21] and its application for imaging of biological tissues was suggested a century later [23]. It began to be developed as a viable medical imaging technique in the middle of 1990s [75, 98].

Some of the mathematical foundations of this imaging modality were originally developed starting in the 1990s for the purposes of the approximation theory $[84,85]($ see $[7,78]$ for extensive reviews of the resulting developments), integral geometry ([48, Chapter 5], [50]), and sonar and radar [27, 86,93].

One can find recent reviews of the physics, biology, and mathematics issues of TAT/PAT 
in $[4,44,45,69,78,99,100,102,114,128,131,133,134,137]$.

TAT/PAT is just one, probably the most advanced at the moment, example of the several recently introduced hybrid imaging methods, which combine different types of radiation to yield high quality of imaging unobtainable by single-radiation modalities (e.g., see [11, 12, $46,79,134]$ for other examples).

\section{Mathematical models of TAT}

In this section, we describe the commonly accepted mathematical model of the TAT procedure and the main mathematical problems that need to be addressed. Since for all our purposes PAT results in the same mathematical model (although the biological features that TAT and PAT detect are different; see details in the chapter [15]), we will refer to TAT only.

\subsection{Point detectors and the wave equation model}

We will mainly assume that point-like omni-directional ultrasound transducers, located throughout an observation (acquisition) surface $S$, are used to detect the values of the pressure $p(y, t)$, where $y \in S$ is a detector location and $t \geq 0$ is the time of the observation. We also denote by $c(x)$ the speed of sound at a location $x$. Then, it has been argued, that the following model describes correctly the propagating pressure wave $p(x, t)$ generated during the TAT procedure (e.g., [15,33, 127, 132, 135]):

$$
\left\{\begin{array}{l}
p_{t t}=c^{2}(x) \Delta_{x} p, \quad t \geq 0, x \in \mathbb{R}^{3} \\
p(x, 0)=f(x), p_{t}(x, 0)=0 .
\end{array}\right.
$$

Here $f(x)$ is the initial value of the acoustic pressure, which one needs to find in order to create the TAT image. In the case of a closed acquisition surface $S$, we will denote by $\Omega$ the interior domain it bounds. Notice that in TAT the function $f(x)$ is naturally supported inside $\Omega$. We will see that this assumption about the support of $f$ sometimes becomes crucial for the feasibility of reconstruction, although some issues can be resolved even if $f$ has non-zero parts outside the acquisition surface.

The data obtained by the point detectors located on a surface $S$ are represented by the function

$$
g(y, t):=p(y, t) \text { for } y \in S, t \geq 0 .
$$

Fig. 2 illustrates the space-time geometry of (1).

We will incorporate the measured data $g$ into the system (1), rewriting it as follows:

$$
\left\{\begin{array}{l}
p_{t t}=c^{2}(x) \Delta_{x} p, \quad t \geq 0, x \in \mathbb{R}^{3} \\
p(x, 0)=f(x), p_{t}(x, 0)=0 \\
\left.p\right|_{S}=g(y, t), \quad(y, t) \in S \times \mathbb{R}^{+} .
\end{array}\right.
$$

Thus, the goal in TAT/PAT is to find, using the data $g(y, t)$ measured by transducers, the initial value $f(x)$ at $t=0$ of the solution $p(x, t)$ of $(3)$.

We will use the following notation: 


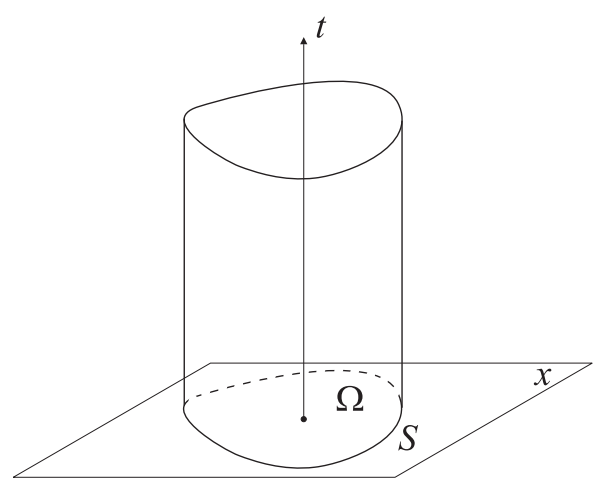

Figure 2: The observation surface $S$ and the domain $\Omega$ containing the object to be imaged.

Definition 1. We will denote by $\mathcal{W}$ the forward operator

$$
\mathcal{W}: f(x) \mapsto g(y, t),
$$

where $f$ and $g$ are described in (3).

\section{Remark 2.}

- The reader should notice that if a different type of detectors is used, the system (1) stays intact, while the measured data will be represented differently from (2) (see Section 2.4). This will correspondingly influence the reconstruction procedures.

- We can consider the same problem in the space $\mathbb{R}^{n}$ of any dimension, not just in $3 D$. This is not merely a mathematical abstraction. Indeed, in the case of the so called integrating line detectors (Section 2.4), one deals with the $2 D$ situation.

\subsection{Acoustically homogeneous media and spherical means}

If the medium being imaged is acoustically homogeneous (i.e., $c(x)$ equals to a constant, which we will assume to be equal to 1 in appropriate units), as it is approximately the case in breast imaging, one deals with the constant coefficient wave equation problem

$$
\left\{\begin{array}{l}
p_{t t}=\Delta_{x} p, \quad t \geq 0, x \in \mathbb{R}^{3} \\
p(x, 0)=f(x), p_{t}(x, 0)=0 \\
\left.p\right|_{S}=g(y, t), \quad(y, t) \in S \times \mathbb{R}^{+} .
\end{array}\right.
$$

In this case, the well known Poisson-Kirchhoff formulas [30, Ch. VI, Section 13.2, Formula (15)] for the solution of the wave equation gives in $3 D$ :

$$
p(x, t)=a \frac{\partial}{\partial t}(t(R f)(x, t))
$$

where

$$
(R f)(x, r):=\frac{1}{4 \pi} \int_{|y|=1} f(x+r y) d A(y)
$$


is the spherical mean operator applied to the function $f(x), d A$ is the standard area element on the unit sphere in $\mathbb{R}^{3}$, and $a$ is a constant. (Versions in all dimensions are known, see (16) and (15).) One can derive from here that knowledge of the function $g(x, t)$ for $x \in S$ and all $t \geq 0$ is equivalent to knowing the spherical mean $R f(x, t)$ of the function $f$ for any points $x \in S$ and any $t \geq 0$. One thus needs to study the spherical mean operator $R: f \rightarrow R f$, or, more precisely, its restriction to the points $x \in S$ only, which we will denote by $\mathcal{M}$ :

$$
\mathcal{M} f(x, t):=\frac{1}{4 \pi} \int_{|y|=1} f(x+t y) d A(y), \quad x \in S, t \geq 0 .
$$

Due to the connection between the spherical mean operator and the wave equation, one can choose to work with the former, and in fact many works on TAT do so. The spherical mean operator $\mathcal{M}$ resembles the classical Radon transform, the common tool of computed tomography $[73,88,89]$, which integrates functions over planes rather than spheres. This analogy with Radon transform, although often purely ideological, rather than technical, provides important intuition and frequently points in reasonable directions of study. However, when the medium cannot be assumed to be acoustically homogeneous, and thus $c(x)$ is not constant, the relation between TAT and integral geometric transforms, such as Radon transform or spherical mean, to a large extent breaks down, and thus one has to work with the wave equation directly.

In what follows, we will address both models of TAT (the PDE model and the integral geometry model) and thus will deal with both forward operators $\mathcal{W}$ and $\mathcal{M}$.

\subsection{Main mathematical problems arising in TAT}

We now formulate a list of problems related to TAT which will be addressed in detail in the rest of the article. (This list is more or less standard for a tomographic imaging method.)

Sufficiency of the data. The first natural question to ask is: Is the data collected on the observation surface $S$ sufficient for the unique reconstruction of the initial pressure $f(x)(3)$ ? In other words, is the kernel of the forward operator $\mathcal{W}$ zero? Or, to put it differently, for which sets $S \in \mathbb{R}^{3}$ the data collected by transducers placed along $S$ determines $f$ uniquely? Yet another interpretation of this question is through observability of solutions of the wave equation on the set $S$ : does observation on $S$ of a solution of the problem (1) determine the solution uniquely?

When the speed of sound is constant, and thus the spherical mean model applies, the equivalent question is whether the operator $\mathcal{M}$ has zero kernel on an appropriate class of functions (say, continuous functions with compact support)

As it is explained in [7], the choice of precise conditions on the local function class, such as continuity, is of no importance for the answer to the uniqueness question, while behavior at infinity (e.g., compactness of support) is. So, without loss of generality, when discussing uniqueness, one can assume $f(x)$ in $(3)$ to be infinitely differentiable.

Inversion formulas and algorithms. Since a practitioner needs to see the actual tomogram, rather than just know its existence, the next natural question arises: If uniqueness the data collected on $S$ is established, what are the actual inversion formulas or 
algorithms? Here again one can work with smooth functions, in the end extending the formulas by continuity to a wider class.

Stability of reconstruction. If we can invert the transform and reconstruct $f$ from the data $g$, how stable is the inversion? The measured data are unavoidably corrupted by errors, and stability means that small errors in the data lead to only small errors in the reconstructed tomogram.

Incomplete data problems. What happens if the data is "incomplete," for instance if one can only partially surround the object by transducers? Does this lead to any specific deterioration in the tomogram, and if yes, to what kind of deterioration?

\section{Range descriptions.}

The next question is known to be important in analysis of tomographic problems: What is the range of the forward operator $\mathcal{W}: f \mapsto g$ that maps the unknown function $f$ to the measured data $g$ ? In other words, what is the space of all possible "ideal" data $g(t, y)$ collected on the surface $S$ ? In the constant speed of sound case, this is equivalent to the question of describing the range of the spherical mean operator $\mathcal{M}$ in appropriate function spaces. Such ranges often have infinite co-dimensions, and the importance of knowing the range of Radon type transforms for analyzing problems of tomography is well known. For instance, such information is used to improve inversion algorithms, complete incomplete data, discover and compensate for certain data errors, etc. (e.g., $[35,47-49,63-65,76,88,89,102]$ and references therein). In TAT, range descriptions are also closely connected with the speed of sound determination problem listed next (see Section 3.6 for a discussion of this connection).

Speed of sound reconstruction. As the reader can expect, reconstruction procedures require the knowledge of the speed of sound $c(x)$. Thus, the problem arises of the recovery of $c(x)$ either from an additional scan, or (preferably) from the same TAT data.

\subsection{Variations on the theme: planar, linear, and circular integrat- ing detectors}

In the described above most basic and well-studied version of TAT, one utilizes point-like broadband transducers to measure the acoustic wave on a surface surrounding the object of interest. The corresponding mathematical model is described by the system (3). In practice, the transducers cannot be made small enough, since smaller detectors yield weaker signals resulting in low signal-to-noise ratios. Smaller transducers are also more difficult to manufacture.

Since finite size of the transducers limits the resolution of the reconstructed images, researchers have been trying to design alternative acquisition schemes using receivers that are very thin but long or wide. Such are $2 D$ planar detectors $[25,56]$ and $1 D$ linear and circular $[26,52,106,142]$ detectors.

We will assume throughout this section that the speed of sound $c(x)$ is constant and equal to 1 . 
Planar detectors are made from a thin piezoelectric polymer film glued onto a flat substrate (see, for example [109]). Let us assume that the object is contained within the sphere of radius $R$. If the diameter of the planar detector is sufficiently large (see [109] for details), it can be assumed to be infinite. The mathematical model of such an acquisition technique is no longer described by (3). Let us define the detector plane $\Pi(s, \omega)$ by equation $x \cdot \omega=s$, where $\omega$ is the unit normal to the plane and $s$ is the (signed) distance from the origin to the plane. Then, while the propagation of acoustic waves is still modeled by (1), the measured data $g_{\text {planar }}(s, t, \omega)$ (up to a constant factor which we will, for simplicity, assume to be equal to 1) can be represented by the following integral:

$$
g_{\text {planar }}(s, \omega, t)=\int_{\Pi(s, \omega)} p(x, t) d A(x)
$$

where $d A(x)$ is the surface measure on the plane. Obviously,

$$
g_{\text {planar }}(s, \omega, 0)=\int_{\Pi(s, \omega)} p(x, 0) d A(x)=\int_{\Pi(s, \omega)} f(x) d A(x) \equiv F(s, \omega),
$$

i.e. the value of $g$ at $t=0$ coincides with the integral $F(s, \omega)$ of the initial pressure $f(x)$ over the plane $\Pi(s, \omega)$ orthogonal to $\omega$.

One can show $[25,56]$ that for a fixed $\omega$, function $g_{\text {planar }}(s, \omega, t)$ is the solution to $1 D$ wave equation

$$
\frac{\partial^{2} g}{\partial s^{2}}=\frac{\partial^{2} g}{\partial t^{2}}
$$

and thus

$$
\begin{aligned}
g_{\text {planar }}(s, \omega, t) & =\frac{1}{2}\left[g_{\text {planar }}(s, \omega, s-t)+g_{\text {planar }}(s, \omega, s+t)\right] \\
& =\frac{1}{2}[F(s+t, \omega)+F(s-t, \omega)] .
\end{aligned}
$$

Since the detector can only be placed outside the object, i.e. $s \geq R$, the term $F(s+t, \omega)$ vanishes, and one obtains

$$
g_{\text {planar }}(s, \omega, t)=F(s-t, \omega) .
$$

In other words, by measuring $g_{\text {planar }}(s, \omega, t)$, one can obtain values of the planar integrals of $f(x)$. If, as proposed in $[25,56]$, one conducts measurements for all planes tangent to the upper half-sphere of radius $R$ (i.e. $s=R, \omega \in S_{+}^{2}$ ), then the resulting data yield all values of the standard Radon transform of $f(x)$. Now the reconstruction can be carried out using one of the many known inversion algorithms for the latter transform (see $[73,88,89]$ ).

Linear detectors are based on optical detection of acoustic signal. Some of the proposed optical detection schemes utilize as the sensitive element a thin straight optical fiber in combination with Fabry-Perot interferometer $[26,52]$. Changes of acoustic pressure on the fiber change (proportionally) its length; this elongation, in turn, is detected by interferometer. A similar idea is used in [106]; in this work the role of a sensitive element is played by a laser beam passing through the water in which the object of interest is submerged, and thus the 
measurement does not perturb the acoustic wave. In both cases, the length of the sensitive element exceeds the size of the object, while the diameter of the fiber (or of the laser beam) can be made extremely small (see [109] for a detailed discussion), which removes restrictions on resolution one can achieve in the images.

Let us assume that the fiber (or laser beam) is aligned along the line $l\left(s_{1}, s_{2}, \omega_{1}, \omega_{2}\right)=$ $\left\{x \mid x=s_{1} \omega_{1}+s_{2} \omega_{2}+s \omega\right\}$, where vectors $\omega_{1}, \omega_{2}$, and $\omega$ form an ortho-normal basis in $\mathbb{R}^{3}$. Then the measured quantities $g_{\text {linear }}\left(s_{1}, s_{2}, \omega_{1}, \omega_{2}, t\right)$ are equal (up to a constant factor which, we will assume, equals to 1 ) to the following line integral:

$$
g_{\text {linear }}\left(s_{1}, s_{2}, \omega_{1}, \omega_{2}, t\right)=\int_{\mathbb{R}^{1}} p\left(s_{1} \omega_{1}+s_{2} \omega_{2}+s \omega, t\right) d s .
$$

Similarly to the case of planar detection, one can show $[26,52,106]$, that for fixed vectors $\omega_{1}, \omega_{2}$ the measurements $g_{\text {linear }}\left(s_{1}, s_{2}, \omega_{1}, \omega_{2}, t\right)$ satisfy the $2 D$ wave equation

$$
\frac{\partial^{2} g}{\partial s_{1}^{2}}+\frac{\partial^{2} g}{\partial s_{2}^{2}}=\frac{\partial^{2} g}{\partial t^{2}}
$$

The initial values $g_{\text {linear }}\left(s_{1}, s_{2}, \omega_{1}, \omega_{2}, 0\right)$ coincide with the line integrals of $f(x)$ along lines $l\left(s_{1}, s_{2}, \omega_{1}, \omega_{2}\right)$. Suppose one makes measurements for all values of $s_{1}(\tau), s_{2}(\tau)$ corresponding to a curve $\gamma=\left\{x \mid x=s_{1}(\tau) \omega_{1}+s_{2}(\tau) \omega_{2}, \tau_{0} \leq \tau \leq \tau_{1}\right\}$ lying in the plane spanned by $\omega_{1}, \omega_{2}$. Then one can try to reconstruct the initial value of $g$ from the values of $g$ on $\gamma$. This problem is a $2 D$ version of (3) and thus the known algorithms (see Section 4) are applicable.

In order to complete the reconstruction from data obtained using line detectors, the measurements should be repeated with different directions of $\omega$. For each value of $\omega$ the $2 D$ problem is solved; the solutions of these problems yield values of line integrals of $f(x)$. If this is done for all values of $\omega$ lying on a half circle, the set of the recovered line integrals of $f(x)$ is sufficient for reconstructing this function. Such a reconstruction represents the inversion of the well known in tomography X-ray transform. The corresponding theory and algorithms can be found, for instance, in [73, 88, 89].

Finally, the use of circular integrating detectors was considered in [142]. Such a detector can be made out of optical fiber combined with an interferometer. In [142], a closed form solution of the corresponding inverse problem is found. However, this approach is very new and neither numerical examples, nor reconstructions from real data have been obtained yet.

\section{Mathematical analysis of the problem}

In this section, we will address most of the issues described in Section 2.3, except the reconstruction algorithms, which will be discussed in Section 4.

\subsection{Uniqueness of reconstruction}

The problem discussed here is the most basic one for tomography: given an acquisition surface $S$ along which we distribute detectors, is the data $g(y, t)$ for $y \in S, t \geq 0$ (see (3)) sufficient for a unique reconstruction of the tomogram $f$ ? A simple counting of variables 
shows that $S$ should be a hyper-surface in the ambient space (i.e., a surface in $\mathbb{R}^{3}$ or a curve in $\mathbb{R}^{2}$ ). As we will see below, although there are some simple counter-examples and remaining open problems, for all practical purposes, the uniqueness problem is positively resolved, and most surfaces $S$ do provide uniqueness. We address this issue for acoustically homogeneous media first and then switch to the variable speed case.

Before doing so, however, we would like to dispel a concern that arises when one looks at the problem of recovering $f$ from $g$ in (3). Namely, an impression might emerge that we consider an initial-boundary value (IBV) problem for the wave equation in the cylinder $\Omega \times \mathbb{R}^{+}$, and the goal is to recover the initial data $f$ from the known boundary data $g$. This is clearly impossible, since according to standard PDE theorems (e.g., [30,36]), one can solve this IBV problem for arbitrary choice of the initial data $f$ and boundary data $g$ (as long as they satisfy simple compatibility conditions, which are fulfilled for instance if $f$ vanishes near $S$ and $g$ vanishes for small $t$, which is the case in TAT). This means that apparently $g$ contains essentially no information about $f$ at all. This argument, however, is flawed, since the wave equation in (3) holds in the whole space, not just in $\Omega$. In other words, $S$ is not a boundary, but rather an observation surface. In particular, considering the wave equation in the exterior of $S$, one can derive that if $f$ is supported inside $\Omega$, the boundary values $g$ of the solution $p$ of (3) also determine the normal derivative of $p$ at $S$ for all positive times. Thus, we in fact have (at least theoretically) the full Cauchy data of the solution $p$ on $S$, which should be sufficient for reconstruction. Another way of addressing this issue is to notice that if the speed of sound is constant, or at least non-trapping (see the definition below in Section 3.1.2), the energy of the solution in any bounded domain (in particular, in $\Omega$ ) must decay in time. The decay when $t \rightarrow \infty$ together with the boundary data $g$ guarantee the uniqueness of solution, and thus uniqueness of recovery $f$.

These arguments, as the reader will see, play a role in understanding reconstruction procedures.

\subsubsection{Acoustically homogeneous media}

We assume here the sound speed $c(x)$ to be constant (in appropriate units, one can choose it to be equal to 1 , which we will do to simplify considerations).

In order to state the first important result on uniqueness, let us recall the system (5), allowing an arbitrary dimension $n$ of the space:

$$
\left\{\begin{array}{l}
p_{t t}=\Delta_{x} p, \quad t \geq 0, x \in \mathbb{R}^{n} \\
p(x, 0)=f(x), p_{t}(x, 0)=0 \\
\left.p\right|_{S}=g(y, t), \quad(y, t) \in S \times \mathbb{R}^{+} .
\end{array}\right.
$$

We introduce the following useful definition:

Definition 3. A set $S$ is said to be uniqueness set, if when used as the acquisition surface, it provides sufficient data for unique reconstruction of the compactly supported tomogram $f$ (i.e., the observed data $g$ in (9) determines uniquely function $f$ ). Otherwise, it is called a non-uniqueness set.

In other words, $S$ is a uniqueness set if the forward operator $\mathcal{W}$ (or, equivalently, $\mathcal{M}$ ) has zero kernel. 
We will start with a very general statement about the acquisition (observation) sets $S$ that provide insufficient information for unique reconstruction of $f$ (see [7] for the proof and references):

Theorem 4. If $S$ is a non-uniqueness set, then there exists a non-zero harmonic polynomial $Q$, which vanishes on $S$.

This theorem implies, in particular, that all "bad" (non-uniqueness) observation sets are algebraic, i.e. have a polynomial vanishing on them. Turning this statement around, we conclude that any set $S$ that is a uniqueness set for harmonic polynomials, is sufficient for unique TAT reconstruction (although, as we will see in Section 3.3, this does not mean practicality of the reconstruction).

The proof of Theorem 4, which the reader can find in $[7,78]$, is not hard and in fact is enlightening, but providing it would lead us too far from the topic of this survey.

We will consider first the case of closed acquisition surfaces, i.e. the ones that completely surround the object to be imaged. We will address the general situation afterwards.

\section{Closed acquisition surfaces $S$}

Theorem 5. ([r]]) If the acquisition surface $S$ is the boundary of bounded domain $\Omega$ (i.e., a closed surface), then it is a uniqueness set. Thus, the observed data $g$ in (9) determines uniquely the sought function $f \in L_{\text {comp }}^{2}\left(\mathbb{R}^{n}\right)$. (The statement holds, even though $f$ is not required to be supported inside $S$.)

Proof: Indeed, since there are no non-zero harmonic functions vanishing on a closed surface $S$, Theorem 4 implies Theorem 5 .

There is, however, another, more intuitive, explanation of why Theorem 5 holds true (although it requires somewhat stronger assumptions, or a more delicate proof than the one indicated below). Namely, since the solution $p$ of (9) has compactly supported initial data, its energy is decaying inside any bounded domain, in particular inside $\Omega$ (see Section 3.1.2 and $[34,67]$ and references therein about local energy decay). On the other hand, if there is non-uniqueness, there exists a non-zero $f$ such that $g(y, t)=0$ for all $y \in S$ and $t$. This means that we can add homogeneous Dirichlet boundary conditions $\left.p\right|_{S}=0$ to (9). But then the standard PDE theorems $[30,36]$ imply that the energy stays constant in $\Omega$. Combination of the two conclusions means that $p$ is zero in $\Omega$ for all times $t$. It is well known [30] that such a solution of the wave equation must be identically zero everywhere, and thus $f=0$.

This energy decay consideration can be extended to some classes of non-compactly supported functions $f$ of the $L^{p}$ classes, leading to the following result of [1]:

Theorem 6. [1] Let $S$ be the boundary of a bounded domain in $\mathbb{R}^{n}$ and $f \in L^{p}\left(\mathbb{R}^{n}\right)$. Then

1. If $p \leq \frac{2 n}{n-1}$ and the spherical mean of $f$ over almost every sphere centered on $S$ is equal to zero, then $f=0$.

2. The previous statement fails when $p>\frac{2 n}{n-1}$ and $S$ is a sphere.

In other words, a closed surface $S$ is a uniqueness set for functions $f \in L^{p}\left(\mathbb{R}^{n}\right)$ when $p \leq \frac{2 n}{n-1}$, and might fail to be such when $p>\frac{2 n}{n-1}$. 
This result shows that the assumption, if not necessarily of compactness of support of $f$, but at least of a sufficiently fast decay of $f$ at infinity, is important for the uniqueness to hold.

\section{General acquisition sets $S$}

Theorems 4 and 5 imply the following useful statement:

Theorem 7. If a set $S$ is not algebraic, or if it contains an open part of a closed analytic surface $\Gamma$, then it is a uniqueness set.

Indeed, the first claim follows immediately from Theorem 4. The second one works out as follows: if an open subset of an analytic surface $\Gamma$ is a non-uniqueness set, then by an analytic continuation type argument (see [7]), one can show that the whole $\Gamma$ is such a set. However, this is impossible, due to Theorem 5.

There are simple examples of non-uniqueness surfaces. Indeed, if $S$ is a plane in $3 D$ (or a line in $2 D$, or a hyperplane in dimension $n$ ) and $f(x)$ in $(3)$ is odd with respect to $S$, then clearly the whole solution of (3) has the same parity and thus vanishes on $S$ for all times $t$. This means that, if one places transducers on a planar $S$, they might register zero signals at all times, while the function $f$ to be reconstructed is not zero. Thus, there is no uniqueness of reconstruction when $S$ is a plane. On the other hand (see [30,72]), if $f$ is supported completely on one side of the plane $S$ (the standard situation in TAT), it is uniquely recoverable from its spherical means centered on $S$, and thus from the observed data $g$.

The question arises what are other "bad" (non-uniqueness) acquisition surfaces than planes. This issue has been resolved in $2 D$ only. Namely, consider a set of $N$ lines on the plane intersecting at a point and forming at this point equal angles. We will call such a figure the Coxeter cross $\Sigma_{N}$ (see Fig. 3). it is easy to construct a compactly supported

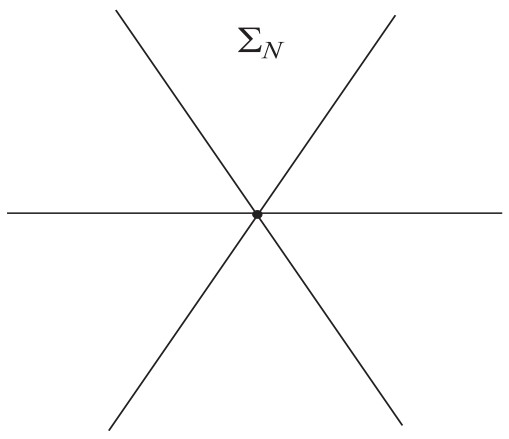

Figure 3: Coxeter cross of $N$ lines.

function that is odd simultaneously with respect of all lines in $\Sigma_{N}$. Thus, a Coxeter cross is also a non-uniqueness set. The following result, conjectured in $[84,85]$ and proven in the full generality in [7], shows that, up to adding finitely many points, this is all that can happen to non-uniqueness sets: 
Theorem 8. [7] A set $S$ in the plane $\mathbb{R}^{2}$ is a non-uniqueness set for compactly supported functions $f$, if and only if it belongs to the union $\Sigma_{N} \bigcup \Phi$ of a Coxeter cross $\Sigma_{N}$ and a finite set of points $\Phi$.

Again, compactness of support is crucial for the proof provided in [7]. There are no other proofs known at the moment of this result (see the corresponding open problem in Section 5). In particular, there is no proven analog of Theorem 6 for non-closed sets $S$ (unless $S$ is an open part of a closed analytic surface).

The $n$-dimensional (in particular, 3D) analog of Theorem 8 has been conjectured [7], but never proven, although some partial advances in this direction have been made in [8,42].

Conjecture 9. A set $S$ in $\mathbb{R}^{n}$ is a non-uniqueness set for compactly supported functions $f$, if and only if it belongs to the union $\Sigma \bigcup \Phi$, where $\Sigma$ is the cone of zeros of a homogeneous (with respect to some point in $\mathbb{R}^{n}$ ) harmonic polynomial, and $\Phi$ is an algebraic sub-set of $\mathbb{R}^{n}$ of dimension at most $n-2$ (see Fig. 4).

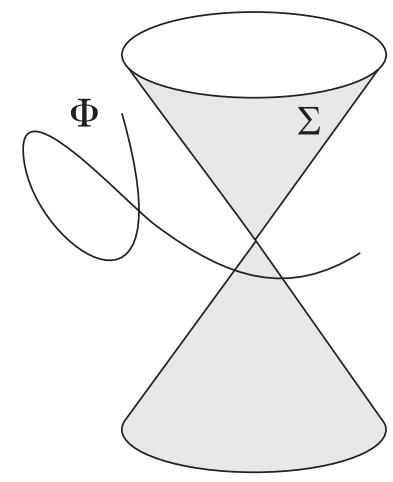

Figure 4: The conjectured structure of a most general non-uniqueness set in $3 D$.

\section{Uniqueness results for a finite observation time}

So far, we have addressed only the question of uniqueness of reconstruction in the nonpractical case of the infinite observation time. There are, however, results that guarantee uniqueness of reconstruction for a finite time of observation. The general idea is that it is sufficient to observe for the time that it takes the geometric rays (see Section 3.1.2) from the interior $\Omega$ of $S$ to reach $S$. In the case of a constant speed, which we will assume to be equal to 1 , the rays are straight and are traversed with the unit speed. This means that if $D$ is the diameter of $\Omega$ (i.e., the maximal distance between two points in the closure of $\Omega$ ), then after time $t=D$, all rays coming from $\Omega$ have left the domain. Thus, one hopes that waiting till time $t=D$ might be sufficient. In fact, due to the specific initial conditions in (3), namely, that the time derivative of the pressure is equal to zero at the initial moment, each singularity of $f$ emanates two rays, and at least one of them will reach $S$ in time not exceeding $D / 2$. And indeed, the following result of [42] holds:

Theorem 10. [42] If $S$ is smooth and closed surface bounding domain $\Omega$ and $D$ is the diameter of $\Omega$, then the TAT data on $S$ collected for the time $0 \leq t \leq 0.5 D$, uniquely determines $f$. 
Notice that a shorter collection time does not guarantee uniqueness. Indeed, if $S$ is a sphere and the observation time is less than $0.5 \mathrm{D}$, due to the finite speed of propagation, no information from a neighborhood of the center can reach $S$ during observation. Thus, values of $f$ in this neighborhood cannot be reconstructed.

\subsubsection{Acoustically inhomogeneous media}

We assume that the speed of sound is strictly positive, $c(x)>c>0$, and such that $c(x)-1$ has compact support, i.e. $c(x)=1$ for large $x$.

\section{Trapping and non-trapping}

We will frequently impose the so-called non-trapping condition on the speed of sound $c(x)$ in $\mathbb{R}^{n}$. To introduce it, let us consider the Hamiltonian system in $\mathbb{R}_{x, \xi}^{2 n}$ with the Hamiltonian $H=\frac{c^{2}(x)}{2}|\xi|^{2}$ :

$$
\left\{\begin{array}{l}
x_{t}^{\prime}=\frac{\partial H}{\partial \xi}=c^{2}(x) \xi \\
\xi_{t}^{\prime}=-\frac{\partial H}{\partial x}=-\frac{1}{2} \nabla\left(c^{2}(x)\right)|\xi|^{2} \\
\left.x\right|_{t=0}=x_{0},\left.\quad \xi\right|_{t=0}=\xi_{0} .
\end{array}\right.
$$

The solutions of this system are called bicharacteristics and their projections into $\mathbb{R}_{x}^{n}$ are rays (or geometric rays).

Definition 11. We say that the speed of sound $c(x)$ satisfies the non-trapping condition, if all rays with $\xi_{0} \neq 0$ tend to infinity when $t \rightarrow \infty$.

The rays that do not tend to infinity, are called trapped.

A simple example, where quite a few rays are trapped, is the radial parabolic sound speed $c(x)=c|x|^{2}$.

It is well known (e.g., [66]) that singularities of solutions of the wave equation are carried by geometric rays. In order to make this statement more precise, we need to recall the notion of a wave front set $W F(u)$ of a distribution $u(x)$ in $\mathbb{R}^{n}$. This set carries detailed information on singularities of $u(x)$.

Definition 12. Distribution $u(x)$ is said to be microlocally smooth near a point $\left(x_{0}, \xi_{0}\right)$, where $x_{0}, \xi_{0} \in \mathbb{R}^{n}$ and $\xi_{0} \neq 0$, if there is a smooth "cut-off" function $\phi(x)$ such that $\phi\left(x_{0}\right) \neq 0$ and that the Fourier transform $\widehat{\phi u}(\xi)$ of the function $\phi(x) u(x)$ decays faster than any power $|\xi|^{-N}$ when $|\xi| \rightarrow \infty$, in directions that are close to the direction of $\xi_{0} .{ }^{1}$

The wave front set $W F(u) \subset \mathbb{R}_{x}^{n} \times\left(\mathbb{R}_{\xi}^{n} \backslash 0\right)$ of $u$ consists of all pairs $\left(x_{0}, \xi_{0}\right)$ such that $u$ is not microlocally smooth near $\left(x_{0}, \xi_{0}\right)$.

In other words, if $\left(x_{0}, \xi_{0}\right) \in W F(u)$, then $u$ is not smooth near $x_{0}$, and the direction of $\xi_{0}$ indicates why it is not: the Fourier transform does not decay well in this direction. For instance, if $u(x)$ consists of two smooth pieces joined non-smoothly across a smooth interface $\Sigma$, then $W F(u)$ can only contain pairs $(x, \xi)$ such that $x \in \Sigma$ and $\xi$ is normal to $\Sigma$ at $x$.

\footnotetext{
${ }^{1}$ We remind the reader that if this Fourier transform decays that way in all directions, then $u(x)$ is smooth (infinitely differentiable) near the point $x_{0}$.
} 
It is known that the wave front sets of solutions of the wave equation propagate with time along the bicharacteristics introduced above. This is a particular instance of a more general fact that applies to general PDEs and can be found in [66,121]. As a result, if after time $T$ all the rays leave the domain $\Omega$ of interest, the solution becomes smooth (infinitely differentiable) inside $\Omega$.

One can find simple introduction to the notions of microlocal analysis, such as the wave front set, for instance in [126], and more advanced versions in [66,121]. Applications of microlocal analysis to integral geometry are discussed in [51,53-55].

The notion of so called local energy decay, which we survey next, is important for the understanding of the non-trapping conditions in TAT.

\section{Local energy decay estimates}

Assuming that the initial data $f(x)(1)$ is compactly supported and the speed $c(x)$ is non-trapping, one can provide the so called local energy decay estimates $[34,129,130]$. Namely, in any bounded domain $\Omega$, the solution $p(x, t)$ of $(1)$ satisfies, for a sufficiently large $T_{0}$ and for any $(k, m)$, the estimate

$$
\left|\frac{\partial^{k+|m|}}{\partial_{t}^{k} \partial_{x}^{m}}\right| \leq C_{k, m} \nu_{k}(t)\|f\|_{L^{2}}, \text { for } x \in \Omega, t>T_{0} .
$$

Here $\nu_{k}(t)=t^{-n+1-k}$ for even $n$ and $\nu_{k}(t)=e^{-\delta t}$ for odd $n$ and some $\delta>0$. Any value $T_{0}$ larger than the diameter of $\Omega$ works in this estimate.

\section{Uniqueness result for non-trapping speeds}

If the speed is non-trapping, the local energy decay allows one to start solving the problem (3) from $t=\infty$, imposing zero conditions at $t=\infty$ and using the measured data $g$ as the boundary conditions. This leads to recovery of the whole solution, and in particular its initial value $f(x)$. As the result, one obtains the following simple uniqueness result of [3]:

Theorem 13. [3] If the speed $c(x)$ is smooth and non-trapping and the acquisition surface $S$ is closed, then the TAT data $g(y, t)$ determines the tomogram $f(x)$ uniquely.

Notice that the statement of the theorem holds even if the support of $f$ is not completely inside of the acquisition surface $S$.

\section{Uniqueness results for finite observation times}

As in the case of constant coefficients, if the speed of sound is non-trapping, appropriately long finite observation time suffices for the uniqueness. Let us denote by $T(\Omega)$ the supremum of the time it takes the ray to reach $S$, over all rays originating in $\Omega$. In particular, if $c(x)$ is trapping, $T(\Omega)$ might be infinite.

Theorem 14. [123] The data $g$ measured till any time $T$ larger than $T(\Omega)$ is sufficient for unique recovery of $f$. 


\subsection{Stability}

By stability of reconstruction of the TAT tomogram $f$ from the measured data $g$ we mean that small variations of $g$ in an appropriate norm lead to small variations of the reconstructed tomogram $f$, also measured by an appropriate norm. In other words, small errors in the data lead to small errors in the reconstruction.

We will try to give the reader a feeling of the general state of affairs with stability, referring to the literature (e.g., $[5,68,78,103,123])$ for further exact details.

We will consider as functional spaces the standard Sobolev spaces $H^{s}$ of smoothness $s$. We will also denote, as before, by $\mathcal{W}$ the operator transforming the unknown $f$ into the data $g$.

Let us recall the notions of Lipschitz and Hölder stability. An even weaker logarithmic stability will not be addressed here. The reader can find discussion of the general stability notions and issues, as applied to inverse problems, in [70].

Definition 15. The operation of reconstructing $f$ from $g$ is said to be Lipschitz stable between the spaces $H^{s_{2}}$ and $H^{s_{1}}$, if the following estimate holds for some constant $C$ :

$$
\|f\|_{H^{s_{1}}} \leq C\|g\|_{H^{s_{2}}} .
$$

The reconstruction is said to be Hölder stable (a weaker concept), if there are constants $s_{1}, s_{2}, s_{3}, C, \mu>0$, and $\delta>0$ such that

$$
\|f\|_{H^{s_{1}}} \leq C\|g\|_{H^{s_{2}}}^{\mu}
$$

for all $f$ such that $\|f\|_{H^{s_{3}}} \leq \delta$.

Stability can be also interpreted in the terms of the singular values $\sigma_{j}$ of the forward operator $f \mapsto g$ in $L^{2}$, which have at most power decay when $j \rightarrow \infty$. The faster is the decay, the more unstable the reconstruction becomes. The problems with singular values decaying faster than any power of $j$ are considered to be extremely unstable. Even worse are the problems with exponential decay of singular values (analytic continuation or solving Cauchy problem for an elliptic operator belong to this class). Again, the book [70] is a good source for finding detailed discussion of such issues.

Consider as an example inversion of the standard in X-ray CT and MRI Radon transform that integrates a function $f$ over hyper-planes in $\mathbb{R}^{n}$. It smoothes function by "adding $(n-1) / 2$ derivatives." Namely, it maps continuously $H^{s}$-functions in $\Omega$ into the Radon projections of class $H^{s+(n-1) / 2}$. Moreover, the reconstruction procedure is Lipshitz stable between these spaces (see [88] for detailed discussion).

One should notice that since the forward mapping is smoothing (it "adds derivatives" to a function), the inversion should produce functions that are less smooth than the data, which is an unstable operation. The rule of thumb is that the stronger is smoothing, the less stable is inversion (this can be rigorously recast in the language of the decay of singular values). Thus, problems that require reconstructing non-smooth functions from infinitely differentiable (or even worse, analytic) data, are extremely unstable (with super-algebraic or exponential decay of singular values correspondingly). This is just a consequence of the standard Sobolev embedding theorems (see, e.g., how this applies in TAT case in [91]). 
In the case of a constant sound speed and the acquisition surface completely surrounding the object, as we have mentioned before, the TAT problem can be recast as inversion of the spherical mean transform $\mathcal{M}$ (see Section 2). Due to analogy between the spheres centered on $S$ and hyperplanes, one suspects that inversion of the spherical mean operator $\mathcal{M}$ is as Lipschitz stable as the inversion of the Radon transform. This indeed is the case, as long as $f$ is supported inside $S$, as can be found in [103]. In the cases when closed form inversion formulas are available (see Section 4.1.1), this stability can also be extracted from them. If the support of $f$ does reach outside, reconstruction of the part of $f$ that is outside is unstable (i.e., is not even Hölder stable, due to the reasons explained in Section 3.3).

In the case of variable non-trapping speed of sound $c(x)$, integral geometry does not apply anymore, and one needs to address the issue using, for instance, time reversal. In this case, stability follows by solving the wave equation in reverse time starting from $t=\infty$, as it is done in [3]. In fact, Lipschitz stability in this case holds for any observation time exceeding $T(\Omega)$ (see [123], where microlocal analysis is used to prove this result).

The bottom line is that TAT reconstruction is sufficiently stable, as long as the speed of sound is non-trapping.

However, trapping speed does cause instability [68]. Indeed, since some of the rays are trapped inside $\Omega$, the information about some singularities never reaches $S$ (no matter for how long one collects the data), and thus, as it is shown in [91], the reconstruction is not even Hölder stable, and the singular values have super-algebraic decay. See also Section 3.3 below for a related discussion.

\subsection{Incomplete data}

In the standard X-ray CT, incompleteness of data arises, for instance, if not all projection angles are accessible, or irradiation of certain regions is avoided, or as in the ROI (region of interest) imaging, only the ROI is irradiated.

It is not that clear what incomplete data means in TAT. Usually one says that one deals with incomplete TAT data, if the acquisition surface does not surround the object of imaging completely. For instance, in breast imaging it is common that only a half-sphere arrangement of transducers is possible. We will see, however, that incomplete data effects in TAT can also arise due to trapping, even if the acquisition surface completely surrounds the object.

The questions addressed here are:

1. Is the collected incomplete data sufficient for unique reconstruction?

2. If yes, does the incompleteness of the data have any effect on stability and quality of the reconstruction?

\subsubsection{Uniqueness of reconstruction}

Uniqueness of reconstruction issues can be considered essentially resolved for incomplete data in TAT, at least in most situations of practical interest. We will briefly survey here some of 
the available results. In what follows, the acquisition surface $S$ is not closed (otherwise the problem is considered to have complete data).

\section{Uniqueness for acoustically homogeneous media}

In this case, Theorem 7 contains some useful sufficient conditions on $S$ that guarantee uniqueness. Microlocal results of $[7,86,122]$, as well as the PDE approach of [42] further applied in [8] provide also some other conditions. We assemble some of these in the following theorem:

Theorem 16. Let $S$ be a non-closed acquisition surface in TAT. Each of the following conditions on $S$ is sufficient for the uniqueness of reconstruction of any compactly supported function $f$ from the TAT data collected on $S$ :

1. Surface $S$ is not algebraic (i.e., there is no non-zero polynomial vanishing on $S$ ).

2. Surface $S$ is a uniqueness set for harmonic polynomials (i.e., there is no non-zero harmonic polynomial vanishing on $S$ ).

3. Surface $S$ contains an open piece of a closed analytic surface $\Gamma$.

4. Surface $S$ contains an open piece of an analytic surface $\Gamma$ separating the space $\mathbb{R}^{n}$ such that $f$ is supported on one side of $\Gamma$.

5. For some point $y \in S$, the function $f$ is supported on one side of the tangent plane $T_{y}$ to $S$ at $y$.

For instance, if the acquisition surface $S$ is just a tiny non-algebraic piece of a surface, data collected on $S$ determines the tomogram $f$ uniquely. However, one realizes that such data is unlikely to be useful for any practical reconstruction. Here the issue of stability of reconstruction kicks in, as it will be discussed in the stability sub-section further down.

\section{Uniqueness for acoustically inhomogeneous media}

In the case of a variable speed of sound, there still are uniqueness theorems for partial data $[123,124]$, e.g.

Theorem 17. [123] Let $S$ be an open part of the boundary $\partial \Omega$ of a strictly convex domain $\Omega$ and the smooth speed of sound equals 1 outside $\Omega$. Then the TAT data collected on $S$ for a time $T>T(\Omega)$ determines uniquely any function $f \in H_{0}^{1}(\Omega)$, whose support does not reach the boundary.

A modification of this result that does not require strict convexity is also available in $[124]$.

While useful uniqueness of reconstruction results exist for incomplete data problems, all such problems are expected to show instability. This issue is discussed in the sub-sections below. This will also lead to a better understanding of incomplete data phenomena in TAT. 


\subsection{2 "Visible" ("audible") singularities}

According to the discussion in Section 3.1.2, the singularities (the points of the wave front set $W F(f)$ of the function $f$ in (3)) are transported with time along the bi-characteristics (10). Thus, in the $x$-space they are transported along the geometric rays. These rays may or may not reach the acquisition surface $S$, which triggers the introduction of the following notion:

Definition 18. A phase space point $\left(x_{0}, \xi_{0}\right)$ is said to be "visible" (sometimes the word "audible" is used instead), if the corresponding ray (see (10)) reaches in finite time the observation surface $S$.

$A$ region $U \subset \mathbb{R}^{n}$ is said to be in the visibility zone, if all points $\left(x_{0}, \xi_{0}\right)$ with $x_{0} \in U$ are visible.

An example of wave propagation through inhomogeneous medium is presented in Figure 5. The open observation surface $S$ in this example consists of the two horizontal and the left vertical sides of the square. Figure 5(a) shows some rays that bend, due to acoustic inhomogeneity, and leave through the opening of the observation surface $S$ (the right side of the square). Fig. 5 (b) presents a flat phantom, whose wavefront set creates these escaping rays, and thus is mostly invisible. Then Fig. 5 (c-f) show the propagation of the corresponding wave front.

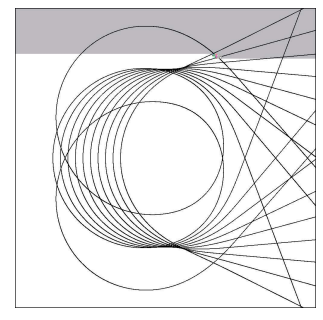

(a)

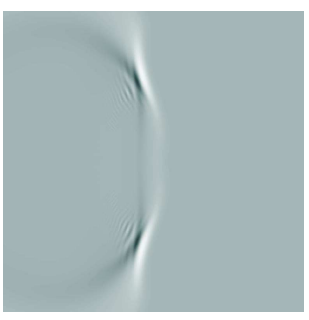

(d)

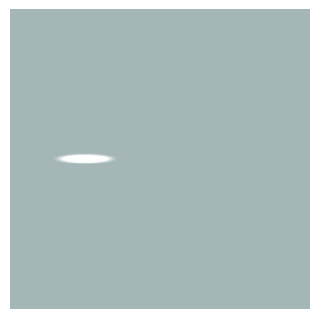

(b)

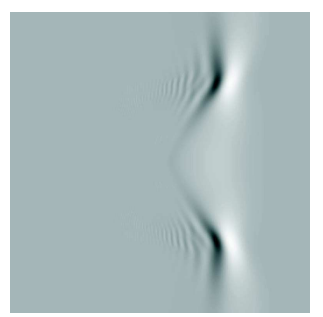

(e)

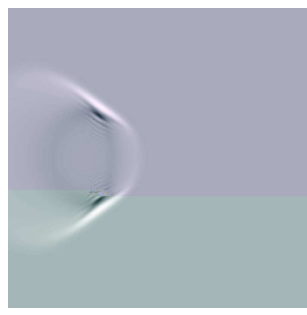

(c)

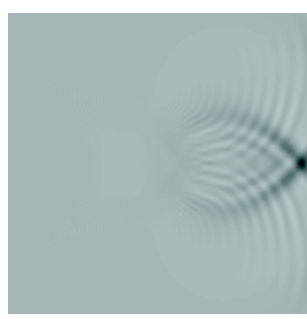

(f)

Figure 5: (a) Some rays starting along the interval $x \in[-0.7,-0.2]$ in the vertical directions escape on the right; (b) a flat phantom with "invisible wavefront"; (c-f)propagation of the flat front: most of the energy of the signal leaves the square domain through the hole on the right.

Since the information about the horizontal boundaries of the phantom escapes, one does not expect to reconstruct it well. Fig. 6 shows two phantoms and their reconstructions 
from the partial data: (a-b) correspond to the vertical flat phantom, whose only invisible singularities are at its ends. One sees essentially good reconstruction, with a little bit of blurring at the endpoints. On the other hand, reconstruction of the horizontal phantom with almost the whole wave front set invisible, does not work. The next Fig. 7 shows a more

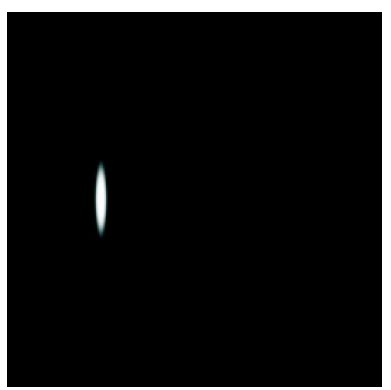

(a)

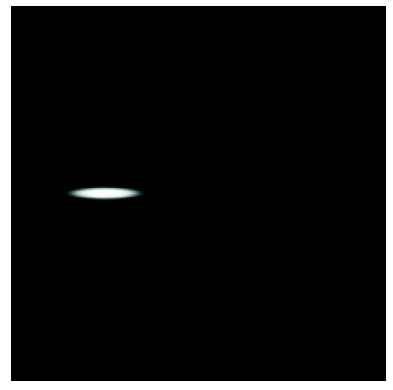

(c)

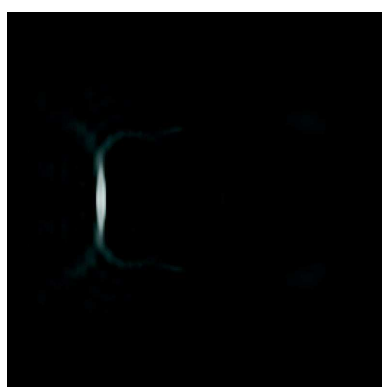

(b)

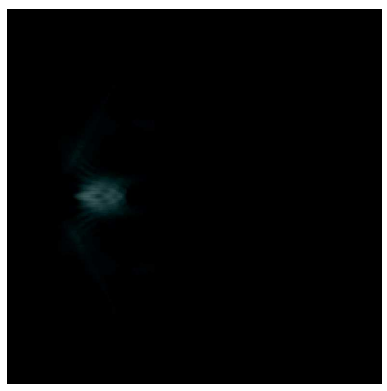

(d)

Figure 6: Reconstruction with the same speed of sound: (a-b) phantom with strong vertical fronts and its reconstruction; (c-d) phantom with strong horizontal fronts and its reconstruction.

complex square phantom, whose singularities corresponding to the horizontal boundaries are invisible, while the vertical boundaries are fine. One sees clearly that the invisible parts have been blurred away. On the other hand, Fig. 11(a) in Section 4 shows that one can

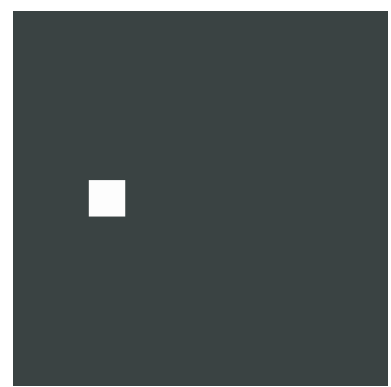

(a)

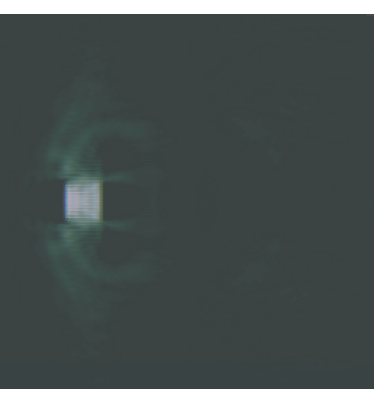

(b)

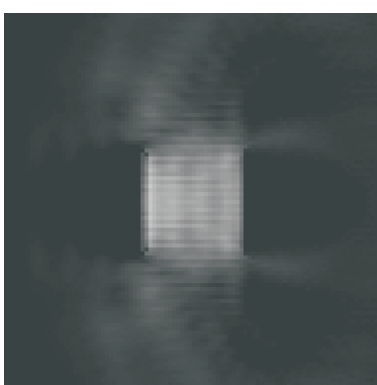

(c)

Figure 7: Reconstruction with the same speed of sound: (a) phantom; (b) its reconstruction; (c) a magnified fragment of (b). 
reconstruct an image without blurring and with correct values, if the image is located in the visibility region. The reconstructed image in this figure is practically indistinguishable from the phantom shown in Figure 10(a).

Remark 19. If $S$ is a closed surface and $x_{0}$ is a point outside of $S$, there is a vector $\xi_{0} \neq 0$ such that $\left(x_{0}, \xi_{0}\right)$ is "invisible." Thus, the visibility zone does not reach outside the closed acquisition surface $S$.

\subsubsection{Stability of reconstruction for incomplete data problems}

In all examples above, uniqueness of reconstruction held, but the images were still blurred. The question arises whether the blurring of "invisible" parts is avoidable (after all, the uniqueness theorems seem to claim that "everything is visible"). The answer to this is, in particular, the following result of [91], which is an analog of similar statements in X-ray tomography:

Theorem 20. [91] If there are invisible points $\left(x_{0}, \xi_{0}\right)$ in $\Omega \times\left(\mathbb{R}_{\xi}^{n} \backslash 0\right)$, then inversion of the forward operator $\mathcal{W}$ is not Hölder stable in any Sobolev spaces. The singular values $\sigma_{j}$ of $\mathcal{W}$ in $L^{2}$ decay super-algebraically.

Thus, having invisible singularities makes the reconstruction severely ill-posed. In particular, according to Remark 19, this theorem implies the following statement:

Corollary 21. Reconstruction of the parts of $f(x)$ supported outside the closed observation surface $S$ is unstable.

On the other hand,

Theorem 22. [123] All visible singularities of $f$ can be reconstructed with Lipschitz stability (in appropriate spaces).

Such a reconstruction of visible singularities can be obtained in many ways, for instance just by replacing the missing data by zeros (with some smoothing along the junctions with the known data, in order to avoid artifact singularities). However, there is no hope for stable recovery of the correct values of $f(x)$, if there are invisible singularities.

\subsection{Discussion of the visibility condition}

\section{Visibility for acoustically homogeneous media}

In the constant speed case, the rays are straight, and thus the visibility condition has a simple test:

Proposition 23. (e.g., [68,140,141]) If the speed is constant, a point $x_{0}$ is in the visible region, if and only if any line passing through $x_{0}$ intersects at least once the acquisition surface $S$ (and thus a detector location).

Figure 8 illustrates this statement. It shows a square phantom and its reconstruction from complete data and from the data collected on the half-circle $S$ surrounding the left half of object. The parts of the interfaces where the normal to the interface does not cross $S$ are blurred. 


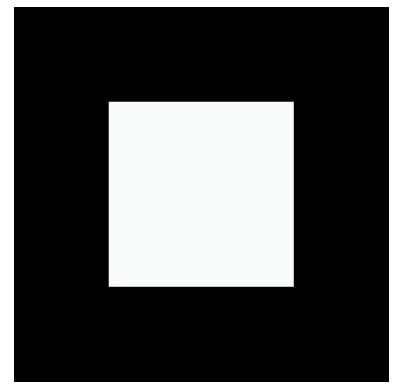

(a)

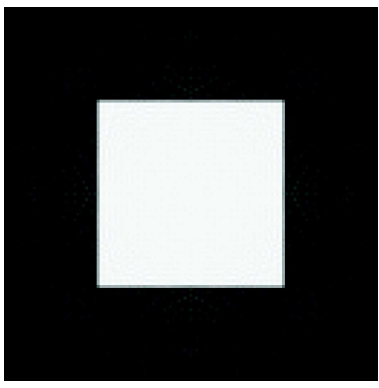

(b)

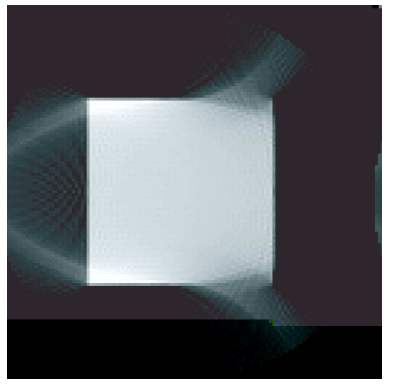

(c)

Figure 8: Reconstruction from incomplete data using closed form inversion formula in $2 D$; detectors are located on the left half circle of radius 1.05 (a) phantom (b) reconstruction from complete data (c) reconstruction from the incomplete data

\section{Visibility for acoustically inhomogeneous media}

When the speed of sound is variable, an analog of Proposition 23 holds, with lines replaced by rays.

Proposition 24. (e.g., $[68,91,123])$ A point $x_{0}$ is in the visible region, if and only if for any $\xi_{0} \neq 0$ at least one of the two geometric rays starting at $\left(x_{0}, \xi_{0}\right)$ and at $\left(x_{0},-\xi_{0}\right)$ (see (10)) intersects the acquisition surface $S$ (and thus a detector location).

The reader can now see an important difference between the acoustically homogeneous and inhomogeneous media. Indeed, even if $S$ surrounds the support of $f$ completely, trapped rays will never find their way to $S$, which will lead, as we know by now, to instabilities and blurring of some interfaces.

Thus, presence of rays trapped inside the acquisition surface creates effects of incomplete data type. This is exemplified in Fig. 9 with a square phantom and its reconstruction shown in the presence of a trapping (parabolic) speed. Notice that the square centered at the center of symmetry of the speed is reconstructed very well (see (d)), since none of the rays carrying its singularities is trapped.

\subsection{Range conditions}

In this section we address the problem of describing the ranges of the forward operators $\mathcal{W}$ (see (4)) and $\mathcal{M}$ (see (8)), the latter in the case of an acoustically homogeneous medium (i.e., for $c=$ const). The ranges of these operators, similarly to the range of the Radon and X-ray transforms (see $[88,89]$ ), are of infinite co-dimensions. This means that ideal data $g$ from a suitable function space satisfy infinitely many mandatory identities. Knowing the range is useful for many theoretical and practical purposes in various types of tomography (reconstruction algorithms, error corrections, incomplete data completion, etc.), and thus this topic has attracted a lot of attention (e.g., [35, 47-49, 63, 64, 76, 80, 88, 89, 102, 119] and references therein).

As we will see in the next section, range descriptions in TAT are also intimately related to recovery of the unknown speed of sound. 


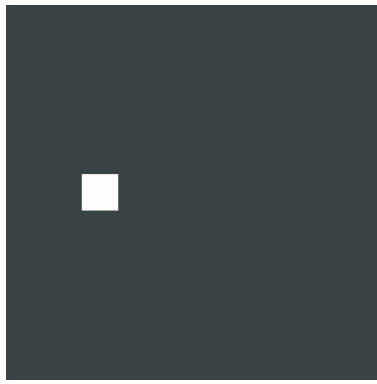

(a)

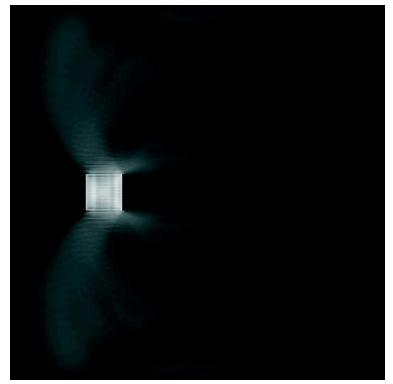

(b)

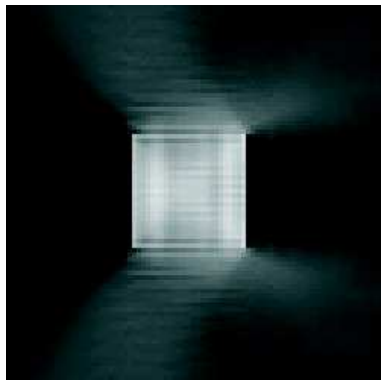

(c)

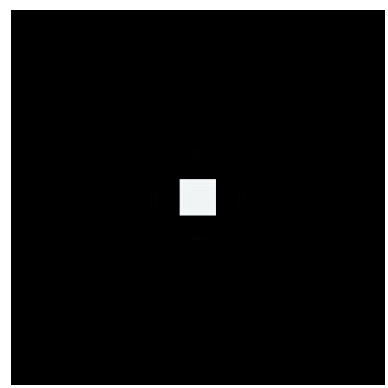

(d)

Figure 9: Reconstruction of a square phantom from full data in the presence of a trapping parabolic speed of sound (the speed is radial with respect to the center of the picture): (a) an off-center phantom; (b) its reconstruction; (c) a magnified fragment of (b); (d) reconstruction of a centered square phantom.

We recall $[47,48,63,88]$ that for the standard Radon transform

$$
f(x) \rightarrow g(s, \omega)=\int_{x \cdot \omega=s} f(x) d x,|\omega|=1,
$$

where $f$ is assumed to be smooth and supported in the unit ball $B=\{x|| x \mid \leq 1\}$, the range conditions on $g(s, \omega)$ are:

1. smoothness and support: $g \in C_{0}^{\infty}([-1,1] \times \mathcal{S})$, where $\mathcal{S}$ is the unit sphere of vectors $\omega$

2. evenness: $g(-s,-\omega)=g(s, \omega)$,

3. moment conditions: for any integer $k \geq 0$, the $k$ th moment

$$
G_{k}(\omega)=\int_{-\infty}^{\infty} s^{k} g(\omega, s) d s
$$

extends from the unit sphere $\mathcal{S}$ to a homogeneous polynomial of degree $k$ in $\omega$.

The seemingly "trivial" evenness condition is sometimes the hardest to generalize to other transforms of Radon type, while it is often easier to find analogs of the moment conditions. This is exactly what happens in TAT.

For the operators $\mathcal{W}, \mathcal{M}$ in TAT, some sets of range conditions of the moment type had been discovered over the years $[7,84,85,111]$, but complete range descriptions started to emerge only since $2006[2,4-6,9,43,78]$.

Range descriptions for the more general operator $\mathcal{W}$ are harder to obtain than for $\mathcal{M}$, and complete range descriptions are not known for even dimensions or for the case of the variable speed of sound.

Let us address the case of the spherical mean operator $\mathcal{M}$ first. 


\subsubsection{The range of the spherical mean operator $\mathcal{M}$.}

The support and smoothness conditions are not hard to come up with, at least when $S$ is a sphere. By choosing appropriate length scale, we can assume that the sphere is of radius 1 and centered at the origin, and that the interior domain $\Omega$ is the unit ball $B=\{x|| x \mid=1\}$. If $f$ is smooth and supported inside $B$ (i.e. $f \in C_{0}^{\infty}(B)$ ), then it is clear that the measured data satisfies the following

Smoothness and support conditions:

$$
g \in C_{0}^{\infty}(S \times[0,2])
$$

An analog of the moment conditions for $g(y, r):=\mathcal{M} f$ was implicitly present in $[7,84,85]$ and explicitly formulated as such in [111]:

Moment conditions: for any integer $k \geq 0$, the moment

$$
M_{k}(y)=\int_{0}^{\infty} r^{2 k+d-1} g(y, r) d r
$$

extends from $S$ to an (in general, non-homogeneous) polynomial $Q_{k}(x)$ of degree at most $2 k$.

These two types of conditions happen to be incomplete, i.e. infinitely many others exist. The Radon transform experience suggests to look for an analog of evenness conditions. And indeed, a set of conditions called orthogonality conditions was found in $[5,9,43]$.

Orthogonality conditions: Let $-\lambda_{k}^{2}$ be the eigenvalue of the Laplace operator $\Delta$ in $B$ with zero Dirichlet conditions and $\psi_{k}$ be the corresponding eigenfunctions. Then the following orthogonality condition is satisfied:

$$
\int_{S \times[0,2]} g(x, t) \partial_{\nu} \psi_{\lambda}(x) j_{n / 2-1}(\lambda t) t^{n-1} d x d t=0 .
$$

Here $j_{p}(z)=c_{p} z^{-p} J_{p}(z)$ is the so called spherical Bessel function.

The range descriptions obtained in $2 D$ in [9] and then in general dimension in [5] showed that these three types of conditions completely describe the range of the operator $\mathcal{M}$ on functions $f \in C_{0}^{\infty}(B)$. At the same time, the results of $[5,43]$ showed that the moment conditions can be dropped in odd dimensions. It was then discovered in [2] that the moment conditions can be dropped altogether in any dimension, since they follow from the other two types of conditions:

Theorem 25. [2] Let $S$ be the unit sphere. A function $g(y, t)$ on the cylinder $S \times \mathbb{R}^{+}$can be represented as $\mathcal{M} f$ for some $f \in C_{0}^{\infty}(B)$ if an only if it satisfied the above smoothness and support and orthogonality conditions (12),(14).

The statement also holds in the finite smoothness case, if one replaces the requirements by $f \in H_{0}^{s}(B)$ and $g \in H_{0}^{s+(n-1) / 2}(S \times[0,2])$.

The range of the forward operator $\mathcal{M}$ has not been described when $S$ is not a sphere, but, say, a convex smooth closed surface. The moment and orthogonality conditions hold for any $S$, and appropriate smoothness and support conditions can also been formulated, at 
least in the convex case. However, it has not been proven that they provide the complete range description.

It is quite possible that for non-spherical $S$ the moment conditions might have to be included into the range description.

A different range description of the Fredholm alternative type was developed in [103] (see also [45] for description of this result).

\subsubsection{The range of the forward operator $\mathcal{W}$.}

We recall that the operator $\mathcal{W}$ (see (4)) transforms the initial value $f$ in (3) into the observed on $S$ values $g$ of the solution. There exist Kirchhoff-Poisson formulas representing the solution $p$, and thus $g=\mathcal{W} f$ in terms of the spherical means of $f$ (i.e., in terms of $\mathcal{M f}$ ). However, translating the result of Theorem 25 into the language of $\mathcal{W}$ is not straightforward, since in even dimensions these formulas are non-local [30,36] (pp. 682 and 801 correspondingly):

$$
\mathcal{W} f(y, t)=\frac{\sqrt{\pi}}{2 \Gamma(n / 2)}\left(\frac{1}{t} \frac{\partial}{\partial t}\right)^{(n-3) / 2} t^{n-2}(\mathcal{M} f)(y, t), \text { for odd } n .
$$

and

$$
\mathcal{W} f(y, t)=\frac{1}{\Gamma(n / 2)}\left(\frac{1}{t} \frac{\partial}{\partial t}\right)^{(n-2) / 2} \int_{0}^{t} \frac{r^{n-1}(\mathcal{M} f)(y, r)}{\sqrt{t^{2}-r^{2}}} d r \text {, for even } n .
$$

The non-locality of the transformation for even dimensions reflects the absence of Huygens' principle (i.e. absence of sharp rear fronts of waves) in these dimensions; it also causes difficulties in establishing the complete range descriptions. In particular, due to the integration in (16) $\mathcal{M} f(y, t)$ does not vanish for large times $t$ anymore. One can try to use other known operators intertwining the two problems (see [5] and references therein), some of which do preserve vanishing for large values of $t$, but this so far has lead only to very clumsy range descriptions.

However, for odd dimensions, the range description of $\mathcal{W}$ can be obtained. In order to do so, given the TAT data $g(y, t)$, let us introduce an auxiliary time-reversed problem in the cylinder $B \times[0,2]$ :

$$
\left\{\begin{array}{l}
\left.q_{t t}-\Delta q=0 \text { for }(x, t) \in B \times[0,2]\right), \\
q(x, 2)=q_{t}(x, 2)=0 \text { for } x \in B \\
q(y, t)=g(y, t) \text { for }(y, t) \in S \times[0,2]) .
\end{array}\right.
$$

We can now formulate the range description from $[43,45]$ :

Theorem 26. [43, 45] For odd dimensions $n$ and $S$ being the unit sphere, a function $g \in$ $C_{0}^{\infty}(S \times[0,2])$ can be represented as $\mathcal{W} f$ for some $f \in C_{0}^{\infty}(B)$ if and only if the following condition is satisfied:

The solution $q$ of (17) satisfies $q_{t}(x, 0)=0$ for all $x \in B$.

Orthogonality type and Fredholm alternative type range conditions, equivalent to the one in the theorem above, are also provided in $[43,45]$. 


\subsection{Speed of sound reconstruction}

Unsurprisingly, all inversion procedures outlined in Section 4 rely upon the knowledge of the speed of sound $c(x)$. Although often, e.g. in breast imaging, the medium is assumed to be acoustically homogeneous, this is not a good assumption in many other cases. It has been observed (e.g., $[68,71]$ ) that replacing even slightly varying speed of sound with its average value might significantly distort the image; not only the numerical values, but also the shapes of interfaces between the tissues will be reconstructed incorrectly. Thus, the question of estimating $c(x)$ correctly becomes important. One possible approach [71] is to use an additional transmission ultrasound scan to reconstruct the speed beforehand. The question arises of whether one could determine the speed of sound $c(x)$ and the tomogram $f(x)$ (assuming that $f$ is not zero) simultaneously from the TAT data. In fact, one needs only to determine $c(x)$ (without knowing $f$ ), since then inversion procedures of Section 4 would apply to recover $f$.

At the first glance, this seems to be an overly ambitious project. Indeed, if we denote the forward operator $\mathcal{W}$ by $\mathcal{W}_{c}$, to indicate its dependence on the speed of sound $c(x)$, then the problem becomes, given the data $g$, to find both $c$ and $f$ from the equality

$$
\mathcal{W}_{c} f=g .
$$

A similar situation arises in the SPECT emission tomography (see $[76,88,89]$ and references therein), where the role of the speed of sound is played by the unknown attenuation. It is known, however, that in SPECT the attenuation can be recovered for a "generic" $f$.

What is the reason for such a strange situation? It looks like for any $c$ one could solve the equation (18) for an $f$, and thus no information about $c$ is contained in the data $g$. This argument is incorrect for the following reason: the range of the forward operator, as we know already from the previous section, has infinite co-dimension. Thus, this range has a lot of space to "rotate" when $c$ changes. Imagine for an instance that the rotation is so powerful that for different values of $c$ the ranges have only zero (the origin) in common. Then, knowing $g$ in the range, one would know which $c$ it came from. Thus, the problem of recovering the speed of sound from the TAT data is closely related to the range descriptions.

Numerical inversions using algebraic iterative techniques (e.g., $[143,144])$ show that recovering both $c$ and $f$ might be indeed possible.

Unfortunately, very little is known at the moment concerning this problem. Direct usage of range conditions attempted in [68] has lead only to extremely weak and not practically useful results so far. A revealing relation to the transmission eigenvalue problem well known in inverse problems (see [29] for the survey) was recently discovered by D. Finch. Unfortunately, the transmission eigenvalue problem remains still unresolved. However, one can derive from this relation the following (still not too useful for TAT) uniqueness of the speed of sound determination result, due to M. Agranovsky:

Theorem 27. If two speeds satisfy the inequality $c_{1}(x) \geq c_{2}(x)$ for all $x \in \Omega$ and produce for some functions $f_{1}, f_{2}$ the same non-zero TAT data g (i.e., $\mathcal{W} c_{1} f_{1}=g, \mathcal{W} c_{2} f_{2}=g$ ), then $c_{1}(x)=c_{2}(x)$.

It is known [70, Corollary 8.2.3] that if a function $f(x)$ is such that $\Delta f(x) \neq 0$ and for two acoustic speeds $c_{1}(x)$ and $c_{2}(x)$ it produces the same TAT data $g$, then $c_{1}=c_{2}$. 
It is clear that the problem of finding the speed of sound from the TAT data still requires significant analysis.

\section{Reconstruction formulas and procedures}

Numerous formulas, algorithms and procedures for reconstruction of images from TAT measurements have been developed by now. Most of these techniques require the data being collected on a closed surface (closed curve in $2 D$ ) surrounding the object to be imaged. Such methods are discussed in Section 4.1. We review methods that work under the assumption of constant speed of sound in Section 4.1.1. The techniques applicable in the case of the known variable speed of sound are considered in Section 4.1.2. Closed surface measurements cannot always be implemented, since in some practical situations the object cannot be completely surrounded by the detectors. In this case, one has to resort to various approximate reconstruction techniques as discussed in Section 4.2.

\subsection{Full data (closed acquisition surfaces)}

\subsubsection{Constant speed of sound}

When the speed of sound within the tissues is a known constant, the TAT problem can be reformulated (see Section 2) in terms of the values of the spherical means of the initial condition $f(x)$. These means can be easily recovered from the measurements of the acoustic pressure using formulas (15) and (16) (see the discussion in [7]). In this case, image reconstruction becomes equivalent to inverting the spherical mean transform $\mathcal{M}$. Thus, in what follows, we consider the problem of reconstructing a function $f(x)$ supported within the region bounded by a closed surface $S$ from known values of its spherical integrals $g(y, r)$ with centers on $S$ :

$$
g(y, r)=\int_{\mathbb{S}^{n-1}} f(y+r \omega) r^{n-1} d \omega, \quad y \in S,
$$

where $d \omega$ is the standard measure on the unit sphere.

\section{Series solutions for spherical geometry}

The first inversion procedures for the case of closed acquisition surfaces were described in $[94,95]$, where solutions were found for the cases of circular (in 2D) and spherical (in $3 D$ ) surfaces, respectively. These solutions were obtained by the harmonic decomposition of the measured data and of the sought function $f(x)$, followed by equating coefficients of the corresponding Fourier series. In particular, the $2 D$ algorithm of [94] pertains to the case when the detectors are located on a circle of radius $R$. This method is based on the Fourier decomposition of $f$ and $g$ in angular variables:

$$
f(x)=\sum_{-\infty}^{\infty} f_{k}(\rho) e^{i k \varphi}, \quad x=(\rho \cos (\varphi), \rho \sin (\varphi))
$$




$$
g(y(\theta), r)=\sum_{-\infty}^{\infty} g_{k}(r) e^{i k \theta}, \quad y=(R \cos (\theta), R \sin (\theta))
$$

where

$$
\left(\mathcal{H}_{m} u\right)(s)=2 \pi \int_{0}^{\infty} u(t) J_{m}(s t) t d t
$$

is the Hankel transform and $J_{m}(t)$ is the Bessel function. As shown in [94], the Fourier coefficients $f_{k}(\rho)$ can be recovered from the known coefficients $g_{k}(r)$ by the following formula:

$$
f_{k}(\rho)=\mathcal{H}_{m}\left(\frac{1}{J_{k}(\lambda|R|)} \mathcal{H}_{0}\left[\frac{g_{k}(r)}{2 \pi r}\right]\right)
$$

This method requires division of the Hankel transform of the measured data by the Bessel functions $J_{k}$, which have infinitely many zeros. Theoretically, there is no problem: the range conditions (Section 3.5) on the exact data $g$ imply that the Hankel transform $\mathcal{H}_{0}\left[(2 \pi r)^{-1} g_{k}(r)\right]$ has zeros that cancel those in the denominator. However, since the measured data always contain errors, the exact cancelation does not happen, and one needs a sophisticated regularization scheme to guarantee that the error remains bounded.

This difficulty can be avoided (see, e.g. [78]) by replacing the Bessel function $J_{0}$ in the inner Hankel transform by the Hankel function $H_{0}^{(1)}$. This yields the following formula for $f_{k}(\rho)$ :

$$
f_{k}(\rho)=\mathcal{H}_{k}\left(\frac{1}{H_{k}^{(1)}(\lambda|R|)} \int_{0}^{\infty} g_{k}(r) H_{0}^{(1)}(\lambda r) d r\right) .
$$

Unlike $J_{m}$, Hankel functions $H_{m}^{(1)}(t)$ do not have zeros for any real values of $t$, which removes the problems with division by zeros [94]. (A different way of avoiding divisions by zero was found in [62])

This derivation can be repeated in $3 D$, with the exponentials $e^{i k \theta}$ replaced by the spherical harmonics, and with cylindrical Bessel functions replaced by their spherical counterparts. By doing this, one arrives at the Fourier series method of [95] (see also [135]). The use of the Hankel function $H_{0}^{(1)}$ above is similar to the way the spherical Hankel function $h_{0}^{(1)}$ is utilized in [95] to avoid the divisions by zero.

\section{Eigenfunction expansions for a general geometry}

The series methods described in the previous section rely on the separation of variables that occurs only in spherical geometry. A different approach was proposed in [82]. It works for arbitrary closed surfaces, but is practical only for those with explicitly known eigenvalues and eigenfunctions of the Dirichlet Laplacian in the interior. Such surfaces include, in particular, spheres, half-sp heres, cylinders, cubes and parallelepipeds, as well as the surfaces of crystallographic domains.

Let $\lambda_{m}^{2}$ and $u_{m}(x)$ be the eigenvalues and an ortho-normal basis of eigenfunctions of the 
Dirichlet Laplacian $-\Delta$ in the interior $\Omega$ of a closed surface $S$ :

$$
\begin{aligned}
\Delta u_{m}(x)+\lambda_{m}^{2} u_{m}(x) & =0, \quad x \in \Omega, \quad \Omega \subseteq \mathbb{R}^{n}, \\
u_{m}(x) & =0, \quad x \in S, \\
\left\|u_{m}\right\|_{2}^{2} & \equiv \int_{\Omega}\left|u_{m}(x)\right|^{2} d x=1 .
\end{aligned}
$$

As before, one would like to reconstruct a compactly supported function $f(x)$ from the known values of its spherical integrals $g(y, r)$ (see (19)) with centers on $S$. Since $u_{m}(x)$ is the solution of the Dirichlet problem for the Helmholtz equation with zero boundary conditions and the wave number $\lambda_{m}$, this function admits the Helmholtz representation

$$
u_{m}(x)=\int_{S} \Phi_{\lambda_{m}}(|x-y|) \frac{\partial}{\partial n} u_{m}(y) d s(y) \quad x \in \Omega,
$$

where $\Phi_{\lambda_{m}}(|x-y|)$ is a free-space Green's function of the Helmholtz equation (21), and $n$ is the exterior normal to $S$.

The function $f(x)$ can be expanded into the series

$$
\begin{aligned}
f(x) & =\sum_{m=0}^{\infty} \alpha_{m} u_{m}(x), \text { where } \\
\alpha_{m} & =\int_{\Omega} u_{m}(x) f(x) d x .
\end{aligned}
$$

A reconstruction formula for $\alpha_{m}$ (and thus for $f(x)$ ) will result, if one substitutes representation (22) into (23) and interchanges the orders of integration:

$$
\alpha_{m}=\int_{\Omega} u_{m}(x) f(x) d x=\int_{S} I\left(y, \lambda_{m}\right) \frac{\partial}{\partial n} u_{m}(y) d A(x)
$$

where

$$
I(y, \lambda)=\int_{\Omega} \Phi_{\lambda}(|x-y|) f(x) d x=\int_{0}^{\operatorname{diam} \Omega} g(y, r) \Phi_{\lambda}(r) d r .
$$

Now $f(x)$ can be obtained by summing the series (23). This method becomes computationally efficient when the eigenvalues and eigenfunctions are known explicitly, especially if a fast summation formula for the series (23) is available. This is the case for a cubic acquisition surface $S$, when the eigenfunctions are products of sine functions. The resulting $3 D$ reconstruction algorithm is extremely fast and precise (see [82]).

The above method has an interesting property. If the support of the source $f(x)$ extends outside $\Omega$, the algorithm still yields theoretically exact reconstruction of $f(x)$ inside $\Omega$. Indeed, the value of the expression (22) for all $x$ lying outside $\Omega$ is zero. Thus, when one computes (24) for $x \in \mathbb{R}^{n} \backslash \Omega$, values of $f(x)$ are multiplied by zero and do not affect further computation in any way. This feature is shared by the time reversal method (see the corresponding paragraph in Section 4.1.2). The closed form FBP type reconstruction techniques considered in the next sub-section, do not have this property. In other words, 
in presence of a source outside the measurement surface, reconstruction within $\Omega$ will be incorrect.

The reason for this difference is that all currently known closed form FBP-type formulas rely (implicitly or explicitly) on the assumption that the wave propagates outside $S$ in the whole free space and has no sources outside. On the other hand, the eig enfunction expansion method and the time reversal rely only upon the time decay of the wave inside $S$, which is not influenced by $f$ having a part outside $S$.

\section{Closed form inversion formulas}

Closed-form inversion formulas play a special role in tomography. They bring about better theoretical understanding of the problem and frequently serve as starting points for the development of efficient reconstruction algorithms. A well known example of the use of explicit inversion formulas is the so-called filtered backprojection (FBP) algorithm in X-ray tomography, which is derived from one of the inversion formulas for the classical Radon transform (see, for example [73,88]).

The very existence of closed form inversion formulas for TAT had been in doubt, till the first such formulas were obtained in odd dimensions by Finch et al in [42], under the assumption that the acquisition surface $S$ is a sphere. Suppose that the function $f(x)$ is supported within a ball of radius $R$ and that the detectors are located on the surface $S=\partial B$ of this ball. Then some of the formulas obtained in [42] read as follows:

$$
\begin{aligned}
& f(x)=-\frac{1}{8 \pi^{2} R} \Delta_{x} \int_{\partial B} \frac{g(y,|y-x|)}{|y-x|} d A(y), \\
& f(x)=-\left.\frac{1}{8 \pi^{2} R} \int_{\partial B}\left(\frac{1}{r} \frac{\partial^{2}}{\partial r^{2}} g(y, r)\right)\right|_{r=|y-x|} d A(y), \\
& f(x)=-\left.\frac{1}{8 \pi^{2} R} \int_{\partial B}\left(\frac{1}{r} \frac{\partial}{\partial r}\left(r \frac{\partial}{\partial r} \frac{g(y, r)}{r}\right)\right)\right|_{r=|y-x|} d A(y),
\end{aligned}
$$

where $d A(y)$ is the surface measure on $\partial B$ and $g$ represents the values of the spherical integrals (19).

These formulas have a FBP (filtered back-projection) nature. Indeed, differentiation with respect to $r$ in (27) and (28) and the Laplace operator in (26) represent the filtration, while the (weighted) integrals correspond to the backprojection, i.e. integration over the set of spheres passing through the point of interest $x$ and centered on $S$.

The so-called "universal backprojection formula" in 3D was found in [136] (it is also valid for the cylindrical and plane acquisition surfaces, see Section 4.2). In our notation, this formula takes the form

$$
f(x)=\left.\frac{1}{8 \pi^{2}} \operatorname{div} \int_{\partial B} n(y)\left(\frac{1}{r} \frac{\partial}{\partial r} \frac{g(y, r)}{r}\right)\right|_{r=|y-x|} d A(y),
$$


or, equivalently,

$$
f(x)=-\left.\frac{1}{8 \pi^{2}} \int_{\partial B} \frac{\partial}{\partial n}\left(\frac{1}{r} \frac{\partial}{\partial r} \frac{g(y, r)}{r}\right)\right|_{r=|y-x|} d A(y),
$$

where $n(y)$ is the exterior normal vector to $\partial B$. One can show $[4,90,136]$ that formulas $(26)$ through (29) are not equivalent on non-perfect data: the result will differ if these formulas are applied to a function that does not belong to the range of the spherical mean transform $\mathcal{M}$. A family of inversion formulas valid in $\mathbb{R}^{n}$ for arbitrary $n \geq 2$ was found in [81]:

$$
f(x)=\frac{1}{4(2 \pi)^{n-1}} \operatorname{div} \int_{\partial B} n(y) h(y,|x-y|) d A(y),
$$

where

$$
\begin{aligned}
h(y, t) & =\int_{\mathbb{R}^{+}} Y(\lambda t)\left[\int_{0}^{2 R} J(\lambda r) g(y, r) d r-J(\lambda t) \int_{0}^{2 R} Y(\lambda r) g(y, r) d r\right] \lambda^{2 n-3} d \lambda, \\
J(t) & =\frac{J_{n / 2-1}(t)}{t^{n / 2-1}}, \quad Y(t)=\frac{Y_{n / 2-1}(t)}{t^{n / 2-1}},
\end{aligned}
$$

and $J_{n / 2-1}(t)$ and $Y_{n / 2-1}(t)$ are respectively the Bessel and Neumann functions of order $n / 2-1$. In $3 D, J(t)$ and $Y(t)$ are simply $t^{-1} \sin t$ and $t^{-1} \cos t$ and formulas (31) and (32) reduce to $(30)$.

In $2 D$, equation (32) also can be simplified [4], which results in the formula

$$
f(x)=\frac{1}{2 \pi^{2}} \operatorname{div} \int_{\partial B} n(y)\left[\int_{0}^{2 R} g(y, r) \frac{1}{r^{2}-|x-y|^{2}} d r\right] d l(y),
$$

where $\partial B$ now stands for the circle of radius $R$ and $d l(y)$ is the standard arc length.

A different set of closed-form inversion formulas applicable in even dimensions was found in [41]. Formula (34) can be compared to the following inversion formulas from [41]:

$$
f(x)=\frac{1}{2 \pi R} \Delta \int_{\partial B} \int_{0}^{2 R} g(y, r) \log \left(r^{2}-|x-y|^{2}\right) d r d l(y),
$$

or

$$
f(x)=\frac{1}{2 \pi R} \int_{\partial B} \int_{0}^{2 R} \frac{\partial}{\partial r}\left(r \frac{\partial}{\partial r} \frac{g(y, r)}{r}\right) \log \left(r^{2}-|x-y|^{2}\right) d r d l(y) .
$$

Finally, a unified family of inversion formulas was derived in [90]. In our notation, it has the following form:

$$
\begin{aligned}
f(x) & =-\left.\frac{4}{\pi R} \int_{\partial B}\left(\frac{\partial}{\partial t} K_{n}(y, t)\right)\right|_{t=|x-y|} \frac{<y-x, y-\xi>}{|x-y|} d A(y), \\
K_{n}(y, t) & =-\frac{1}{16(2 \pi)^{n-2}} \int_{\mathbb{R}^{+}} \lambda^{2 n-3} Y(\lambda t)\left(\int_{\mathbb{R}^{+}} J(\lambda r) g(y, r) d r\right) d \lambda
\end{aligned}
$$


where $\partial B$ is the surface of a ball in $\mathbb{R}^{n}$ of radius $R$, functions $J$ and $Y$ are as in (33), and $\xi$ is an arbitrary fixed vector. In particular, in $3 D$

$$
J(t)=\sqrt{\frac{2}{\pi}} \frac{\sin t}{t}, J(t)=\sqrt{\frac{2}{\pi}} \frac{\cos t}{t}
$$

and, after simple calculation, the above inversion formula reduces to

$$
f(x)=-\left.\frac{1}{8 \pi^{2} R} \int_{\partial B}\left(\frac{\partial}{\partial r} \frac{1}{r} \frac{\partial}{\partial r} \frac{g(y, r)}{r}\right)\right|_{r=|x-y|} \frac{<y-x, y-\xi>}{|x-y|} d A(y) .
$$

Different choices of vector $\xi$ in the above formula result in different inversion formulas. For example, if $\xi$ is set to zero, the ratio $\frac{\langle y-x, y-\xi\rangle}{|x-y|}$ equals $R \cos \alpha$, where $\alpha$ is the angle between the exterior normal $n(y)$ and the vector $y-x$; when combined with the derivative in $t$ this factor produces the normal derivative, and the inversion formula (38) reduces to (30). On the other hand, the choice of $\xi=x$ in (38) leads to a formula

$$
f(x)=-\left.\frac{1}{8 \pi^{2} R} \int_{\partial B}\left(r \frac{\partial}{\partial r} \frac{1}{r} \frac{\partial}{\partial r} \frac{g(y, r)}{r}\right)\right|_{r=|x-y|} d A(y)
$$

which is reminiscent of formulas (26)-(28).

\section{Greens' formula approach and some symmetry considerations}

Let us suppose for a moment that the acoustic detectors could measure not only the pressure $p(y, t)$ at each point of the acquisition surface $S$, but also the normal derivative $\partial p / \partial n$ on $S$. Then the problem of reconstructing the initial pressure $f(x)$ becomes rather simple. Indeed, one can use the knowledge of the free-space Green's function for the wave equation and invoke the Green's theorem to represent the solution $p(x, t)$ of $(3)$ in the form of integrals over $S$ involving $p(x, t)$ and its normal derivative and the Green's function and its normal derivative. (This can be done in the Fourier or time domains.) This would require infinite observation time, but in $3 D$ the time $T(\Omega)$ will suffice, afte $\mathrm{r}$ which the wave escapes the region of interest (a cut-off also would work approximately in $2 D$. similarly to the timereversal method). This Green's function approach happens to be, explicitly or implicitly, the starting point of all closed form inversions described above. The trick is to rewrite the formula in such a way that the unknown in reality normal derivative $\partial p / \partial n$ disappears from the formula.

This was achieved in [81] by reducing the question to some integrals involving special functions and making the key observation that the integral

$$
I_{\lambda}(x, y)=\int_{\partial B} J(\lambda|x-z|) \frac{\partial}{\partial n} Y(\lambda|y-z|) d A(z), \quad x, y \in B \subset \mathbb{R}^{n}
$$

is a symmetric function of its arguments:

$$
I_{\lambda}(x, y)=I_{\lambda}(y, x) \text { for } x, y \in B \subset \mathbb{R}^{n}
$$


Similarly, the derivation of (37) in [90] employs the symmetry of the integral

$$
K_{\lambda}(x, y)=\int_{\partial B} J(\lambda|x-z|) Y(\lambda|y-z|) d A(z), \quad x, y \in B \subset \mathbb{R}^{n} .
$$

In fact, the symmetry holds for any integral

$$
W_{\lambda}(x, y)=\int_{\partial B} U(\lambda|x-z|) V(\lambda|y-z|) d A(z), \quad x, y \in B \subset \mathbb{R}^{n},
$$

where $U(\lambda|x|)$ and $V(\lambda|x|)$ are any two radial solutions of Helmholtz equation

$$
\Delta u(x)+\lambda^{2} u(x)=0 .
$$

It is straightforward to verify this symmetry when $S$ is a sphere and $B$ is the corresponding ball, and the points $x, y$ lie on the boundary $S$ only, rather than anywhere in $B$. This follows immediately from the rotational symmetry of $S$. The same i s true for the normal derivatives on $S$ of $W_{\lambda}(x, y)$ in $x$ and $y$.

This boundary symmetry happens to imply the needed full symmetry (39) for $x, y \in B$.

Indeed, $W_{\lambda}(x, y)$ is a solution of the Helmholtz equation separately as a function of $x$ and of $y$. Let us introduce a family of solutions $\left\{w_{n}(x)\right\}_{n=0}^{\infty}$ of (40) in $B$, such that the members of this family form an orthonormal basis for all solutions of the latter equation in $B$. For example, the spherical waves, i.e. the products of spherical harmonics and Bessel functions, can serve as such a basis.

Then $W_{\lambda}(x, y)$ can be expanded $\mathrm{n}$ the following series:

$$
W_{\lambda}(x, y)=\sum_{n=0}^{\infty} \sum_{m=0}^{\infty} b_{n, m} w_{m}(y) w_{n}(x)
$$

Since $W_{\lambda}(x, y)$ is a solution to the Helmholtz equation in $\partial B \times \partial B$, coefficients $b_{n, m}$ are completely determined by the boundary values of $W_{\lambda}$. Since the boundary values are symmetric, the coefficients are symmetric, i.e. $b_{n, m}=b_{m, n}$ which by (41) immediately implies $W_{\lambda}(x, y)=W_{\lambda}(y, x)$ for all pairs $(x, y) \in B \times B$.

This consideration extends to infinite cylinders and planes. This explains why the "universal backprojection formula" (30) is valid also for infinite cylinders and planes [136]. Since the sort of symmetry used is shared only by these three surfaces, we believe it is unlikely that a closed-form formula could exist for any other acquisition surface.

\section{Algebraic iterative algorithms}

Iterative algebraic techniques are among the favorite tomographic methods of reconstruction and have been used in CT for quite a while $[73,88,89]$. They amount to discretizing the equation relating the measured data with the unknown source, followed by iterative solution of the resulting linear system. Iterative algebraic reconstruction algorithms frequently produce better images than those obtained by other methods. However, they are notoriously slow. In TAT, they have been used successfully for reconstructions with partial data $([16,17,108])$, see Section 4.2 . 


\section{Parametrix approaches}

Some of the earlier non-iterative reconstruction techniques [75] were of approximate nature. For example, by approximating the integration spheres by their tangent planes at the point of reconstruction and by applying one of the known inversion formulas for the classical Radon transform, one can reconstruct an approximation to the image. Due to the evenness symmetry in the classical Radon projections (see Section 3.5), the normals to the integration planes need only fill a half of a unit sphere, in order to make possible the reconstruction from an open measurement surface. A more sophisticated approach is represented by the so-called "straightening" methods $[115,116]$ based on the approximate reconstruction of the classical Radon projections from the values of the spherical mean transform $\mathcal{M} f$ of the function $f(x)$ in question. These methods yield not a true inversion, but rather what is called in micro-local analysis a parametrix. Application of a parametrix reproduces the function $f$ with an additional, smoother term. In other words, the locations (and often the sizes) of jumps across sharp material interfaces, as well as the whole wave front set $W F(f)$, are reconstructed correctly, while the accuracy of the lower spatial frequencies cannot be guaranteed. (Sometimes, the reconstructed function has a more general form $A f$, where $A$ is an elliptic pseudo-differential operator $[66,121,126]$ of order zero. In this case, the sizes of the jumps across the interfaces might be altered.) Unlike the approximations resulting from the discretization of the exact inversion formulas (in the situations when such formulas are known), the parametrix approximations do not converge, when the discretization of the data is refined and the noise is eliminated. Parametrix reconstructions can be either accepted as approximate images, or used as starting points for iterative algorithms. See [123] for a recent discussion of parametrices.

These methods are closely related to the general scheme proposed in $[22,32]$ for the inversion of the generalized Radon transform with integration over curved manifolds. It reduces the problem to a Fredholm integral equation of the second kind, which is well suited for numerical solution. Such an approach amounts to using a parametrix method as an efficient pre-conditioner for an iterative solver; the convergence of such iterations is much faster than that of algebraic iterative methods.

\section{Numerical implementation and computational examples.}

By discretizing exact formulas presented above, one can easily develop accurate and efficient reconstruction algorithms. The $3 D$ case is especially simple: computation of derivatives in the formulas (26)-(30) and (38) can be easily done, for instance by using finite differences; it is followed by the backprojection (described by the integral over $\partial B$ ), which requires prescribing quadrature weights for quadrature nodes that coincide with the positions of the detectors. The backprojection step is stable; the differentiation is a mildly unstable operation. The sensitivity to noise in measurements across the formulas presented above seems to be roughly the same. It is very similar to that of the widely used FBP algorithm of classical X-ray tomography $[88,89]$. In $2 D$, the implementation is just a little bit harder: the filtration step in formulas (34)-(36) can be reduced to computing two Hilbert transforms (see [78]), which, in turn, can be easily done in the frequency domain.

The number of floating point operations (flops) required by such algorithms is determined

by the slower backprojection step. In $3 D$, if the number of detectors is $m^{2}$ and the size of the reconstruction grid is $m \times m \times m$, the backprojection step (and the whole algorithm) 
will require $O\left(\mathrm{~m}^{5}\right)$ flops. In practical terms this amounts to several hours of computations on a single processor computer for a grid of size $129 \times 129 \times 129$.

In $2 D$, the operation count is just $O\left(\mathrm{~m}^{3}\right)$. As it is discussed in Section 2.4, the $2 D$ problem needs to be solved, when integrating line detectors are used. In this situation, the $2 D$ problem needs to be solved $m$ times in order $\mathrm{t}$ o reconstruct the image, which raises the total operation count to $O\left(m^{4}\right)$ flops.

Figure 10 shows three examples of simulated reconstruction using formula (34). The phantom we use (Figure 10(a)) is a linear combination of several characteristic functions of disks and ellipses. Part (b) illustrates the image reconstruction within the unit circle from 257 equi-spaced projections each containing 129 spherical integrals. The detectors were placed on the concentric circle of radius 1.05. The image shown in Figure 10(c) corresponds to the reconstruction from the simulated noisy data that were obtained by adding to projections values of a random variable scaled so that the $L^{2}$ intensity of the noise was $15 \%$ of the intensity of the signal. Finally, Figure 10(d) shows how application of a smoothing filter (in the frequency domain) suppresses the noise; it also somewhat blurs the edges in the image.

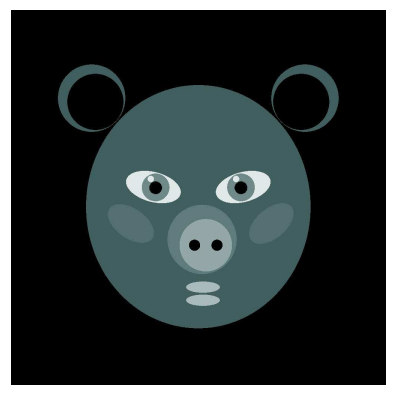

(a)

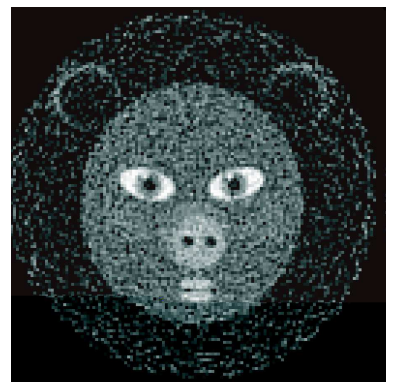

(c)

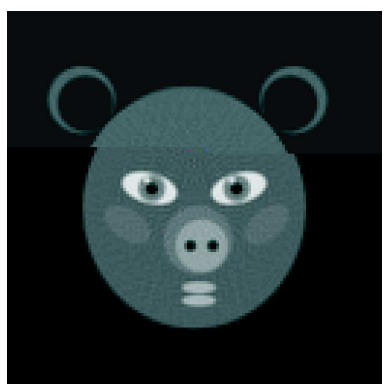

(b)

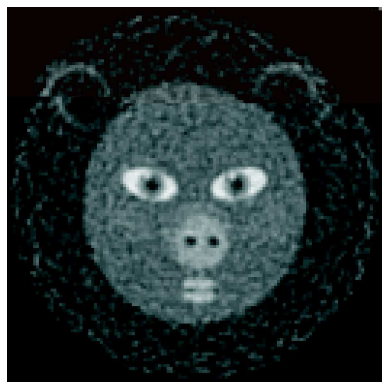

(d)

Figure 10: Example of a reconstruction using formula (34): (a) phantom; (b) reconstruction from accurate data; (c) reconstruction from the data contaminated with $15 \%$ noise; (d) reconstruction from the noisy data with additional smoothing

\subsubsection{Variable speed of sound}

The reconstruction formulas and algorithms described in the previous section work under the assumption that the speed of sound within the region of interest is constant (or at least close to a constant). This assumption, however, is not always realistic - for example, if the region 
of interest contains both soft tissues and bones, the speed of sound will vary significantly. Experiments with numerical and physical phantoms show $[68,71]$ that if acoustic inhomogeneities are not taken into account, the reconstructed image might be severely distorted. Not only the numerical values could be reconstructed incorrectly, but so would the material interface locations and discontinuity magnitudes.

Below we review some of the reconstruction methods that work in acoustically inhomogeneous media. We will assume that the speed of sound $c(x)$ is known, smooth, positive, constant for large $x$, and non-trapping. In practice, a transmission ultrasound scan can be used to reconstruct $c(x)$ prior to thermoacoustic reconstruction, as it is done in [71].

\section{Time reversal}

Let us assume temporarily that the speed of sound $c$ is constant and the spatial dimension is odd. Then Huygens' principle guarantees that the sound wave will leave the region of interest $\Omega$ in time $T=c /(\operatorname{diam} \Omega)$, so that $p(x, t)=0$ for all $x \in \Omega$ and $t \geq T$. Now one can solve the wave equation back in time from $t=T$ to $t=0$ in the domain $\Omega \times[T, 0]$, with zero initial conditions at $T$ and boundary conditions on $S$ provided by the data $g$ collected by the detectors. Then the value of the solution at $t=0$ will coincide with the initial condition $f(x)$ that one seeks to reconstruct. Such a solution of the wave equation is easily obtained numerically by finite difference techniques $[52,68]$. The required number of floating point operations is actually lower than that of methods based on discretized inversion formulas $\left(\mathcal{O}\left(m^{4}\right)\right.$ for time reversal on a grid $m \times m \times m$ in $3 D$ versus $\mathcal{O}\left(m^{5}\right)$ for inversion formulas), which makes this method quite competitive even in the case of constant speed of sound.

Most importantly, however, the method is also applicable if the speed of sound $c(x)$ is variable and/or the spatial dimension is even. In these cases, the Huygens' principle does not hold, and thus the solution to the direct problem will not vanish within $\partial \Omega$ in finite time. However, the solution inside $\Omega$ will decay with time. Under the non-trapping condition, as it is shown in (11) (see $[34,129,130]$ ), the time decay is exponential in odd dimensions, but only algebraic in even-dimensions. Although, in order to obtain theoretically exact reconstruction, one would have to start the time reversal at $T=\infty$, numerical experiments (e.g., [68]) and theoretical estimates [67] show that in practice it is sufficient to start at the values of $T$ when the signal becomes small enough, and to approximate the unknown value of $p(x, T)$ by zero (a more sophisticated cut-off is used in [123], which leads to an equation with a contraction operator). This works $[52,68]$ even in $2 D$ (where decay is the slowest) and in inhomogeneous media. However, when trapping occurs, the "invisible" parts blur away (see Section 3.3 for the discussion).

\section{Eigenfunction expansions.}

An "inversion formula" that reconstructs the initial value $f(x)$ of the solution of the wave equation from values on the measuring surface $S$ can be easily obtained using time reversal and Duhamel's principle [3]. Consider in $\Omega$ the operator $A=-c^{2}(x) \Delta$ with zero Dirichlet conditions on the boundary $S=\partial \Omega$. This operator is self-adjoint, if considered in the weighted space $L^{2}\left(\Omega ; c^{-2}(x)\right)$. Let us denote by $E$ the operator of harmonic extension, which transforms a function $\phi$ on $S$ to a harmonic function on $\Omega$ which coincides with $\phi$ on 
$S$. Then $f$ can be reconstructed [3] from the data $g$ in (3) by the following formula:

$$
f(x)=\left(\left.E g\right|_{t=0}\right)-\int_{0}^{\infty} A^{-\frac{1}{2}} \sin \left(\tau A^{\frac{1}{2}}\right) E\left(g_{t t}\right)(x, \tau) d \tau,
$$

which is valid under the non-trapping condition on $c(x)$. However, due to the involvement of functions of the operator $A$, it is not clear how useful this formula can be.

One natural way to try to implement numerically the formula (42) is to use the eigenfunction expansion of the operator $A$ in $\Omega$ (assuming that such expansion is known). This quickly leads to the following procedure [3]. The function $f(x)$ can be reconstructed inside $\Omega$ from the data $g$ in (3), as the following $L^{2}(B)$-convergent series:

$$
f(x)=\sum_{k} f_{k} \psi_{k}(x)
$$

where the Fourier coefficients $f_{k}$ can be recovered from the data using one of the following formulas:

$$
\begin{gathered}
f_{k}=\lambda_{k}^{-2} g_{k}(0)-\lambda_{k}^{-3} \int_{0}^{\infty} \sin \left(\lambda_{k} t\right) g_{k}^{\prime \prime}(t) d t \\
f_{k}=\lambda_{k}^{-2} g_{k}(0)+\lambda_{k}^{-2} \int_{0}^{\infty} \cos \left(\lambda_{k} t\right) g_{k}^{\prime}(t) d t, \text { or } \\
f_{k}=-\lambda_{k}^{-1} \int_{0}^{\infty} \sin \left(\lambda_{k} t\right) g_{k}(t) d t=-\lambda_{k}^{-1} \int_{0}^{\infty} \int_{S} \sin \left(\lambda_{k} t\right) g(x, t) \overline{\frac{\partial \psi_{k}}{\partial n}(x)} d x d t
\end{gathered}
$$

where

$$
g_{k}(t)=\int_{S} g(x, t) \overline{\frac{\partial \psi_{k}}{\partial n}(x)} d x .
$$

One notices that this is a generalization of the expansion method of [82] discussed in Section 4.1.1 to the case of a variable speed of sound. Unlike the algorithm of [82], the present method does not require the knowledge of the whole space Green's function for $A$ (which is in this case unknown). However, computation of a large set of eigenfunctions and eigenvalues followed by the summation of the series (43) at the nodes of the computational grid may prove to be too time consuming.

It is worthwhile to mention again that the non-trapping condition is crucial for the stability of any TAT reconstruction method in acoustically inhomogeneous media. As it was discussed in Section 3.4, trapping can significantly reduce the quality of reconstruction. It is, however, most probable that trapping does not occur much in biological objects.

\subsection{Partial (incomplete) data}

Reconstruction formulas and algorithms of the previous sections work under the assumption that the acoustic signal is measured by detectors covering a closed surface $S$ that surrounds completely the object of interest. However, in many practical applications of TAT, detectors can be placed only on a certain part of the surrounding surface. Such is the case, for 
example, when TAT is used for breast screening - one of the most promising applications of this modality. Thus, one needs methods and algorithms capable of accurate reconstruction of images from partial (incomplete) data, i.e. from the measurements made on open surfaces (or open curves in 2D).

Most exact inversion formulas and methods discussed above are based (explicitly or implicitly) on some sort of the Green's formula, Helmholtz representation, or eigenfunction decomposition for closed surfaces, and thus they cannot be extended to the case of partial data. The methods that do work in this situation rely on approximation techniques, as discussed below.

\subsubsection{Constant speed of sound}

Even the case of an acoustically homogeneous medium is quite challenging when reconstruction needs to be done from partial data (i.e., when the acquisition surface $S$ is not closed). As it was discussed in Section 3.3, if the detectors located around the object in such a way that the "visibility" condition is not satisfied, accurate reconstruction is impossible: the "invisible" interfaces will be smoothed out in the reconstructed image. On the other hand, if the visibility condition is satisfied, the reconstruction is only mildly unstable (similarly to the inversion of the classic Radon transform) [103,123]. If, in addition, the uniqueness of reconstruction from partial data is guaranteed (which is usually the case, see Section 3.3.1), one can hope to be able to develop an algorithm that would reconstruct quality images.

Special cases of open acquisition surfaces are a plane or an infinite cylinder, for which exact inversion formulas are known (see, for example, [18, 40, 48, 92, 138] for the plane and [139] or for a cylinder). Of course, the plane or a cylinder would have to be truncated in any practical measurements. The resulting acquisition geometry will not satisfy the visibility condition, and material interfaces whose normals do not intersect the acquisition surface will be blurred.

Iterative algebraic techniques (see the corresponding paragraph in Section 4.1.1) were among the first methods successfully used for reconstruction from surfaces only partially surrounding the object (e.g., [16,17, 108]). As it is mentioned in Section 4.1.1, such methods are very slow. For example, reconstructions in [17] required the use of a cluster of computers and took 100 iterations to converge.

Parametrix type reconstructions in the partial data case were proposed in [19]. A couple of different parametrix-type algorithms were proposed in [105,107]. They are based on applying one of the exact inversion formulas for full circular acquisition to the available partial data, with zero-filled missing data and some correction factors. Namely, since the missing data is replaced by zeros, each line passing through a node of the reconstruction grid will be tangent either to one or to two circles of integration. Therefore some directions during the backprojection step will be represented twice, and some only once. This, in turn, will cause some interfaces to appear twice stronger then they should be. The use of weight factors was proposed in $[105,107]$ in order to partially compensate for this distortion. In particular, in [105] smooth weight factors (depending on a reconstruction point) are assigned to each detector in such a way that the total weight for each direction is exactly one. This method is not exact; the error is described by a certain smoothing operator. However, the singularities (or jumps) in the image will be reconstructed correctly. As shown by numerical examples in 
[105], such a correction visually significantly improves the reconstruction. Moreover, iterative refinement is proposed in $[105,107]$ to further improve the image, and it is shown to work well in numerical experiments.

Returning to non-iterative techniques, one should mention an interesting attempt made in $[111,112]$ ) to generate the missing data using the moment range conditions for $\mathcal{M}$ (see Section 3.5). The resulting algorithm, however, does not seem to recover the values well; although, as expected, it reconstructs all visible singularities.

An accurate $2 D$ non-iterative algorithm for reconstruction from data measured on an open curve $S$ was proposed in [83]. It is based on pre-computing approximations of plane waves in the region of interest $\Omega$ by the single layer potentials of the form

$$
\int_{S} Z(\lambda|y-x|) \rho(y) d l(y),
$$

where $\rho(y)$ is the density of the potential, which needs to be chosen appropriately, $d l(y)$ is the standard arc length, and $Z(t)$ is either the Bessel function $J_{0}(t)$, or the Neumann function $Y_{0}(t)$. Namely, for a fixed $\xi$ one finds numerically the densities $\rho_{\xi, J}(y)$ and $\rho_{\xi, Y}(y)$ of the potentials

$$
\begin{aligned}
W_{J}\left(x, \rho_{\xi, J}\right) & =\int_{S} J_{0}(\lambda|y-x|) \rho_{\xi, J}(y) d l(y), \\
W_{Y}\left(x, \rho_{\xi, Y}\right) & =\int_{S} Y_{0}(\lambda|y-x|) \rho_{\xi, Y}(y) d l(y),
\end{aligned}
$$

where $\lambda=|\xi|$, such that

$$
W_{J}\left(x, \rho_{\xi, J}\right)+W_{Y}\left(x, \rho_{\xi, Y}\right) \approx \exp (-i \xi \cdot x) \text { for all } x \in \Omega .
$$

Obtaining such approximations is not trivial. One can show that exact equality in (47) cannot be achieved, due to different behavior at infinity of the plane wave and the approximating single-layer potentials. However, as shown by numerical examples in [83], if each point in $\Omega$ is "visible" from $S$, very accurate approximations can be obtained, while keeping the densities $\rho_{\xi, J}$ and $\rho_{\xi, Y}$ under certain control.

Once the densities $\rho_{\xi, J}$ and $\rho_{\xi, Y}$ have been found for all $\xi$, function $f(x)$ can be easily reconstructed. Indeed, for the Fourier transform $\hat{f}(\xi)$ of $f(x)$

$$
\hat{f}(\xi)=\frac{1}{2 \pi} \int_{\Omega} f(x) \exp (-i \xi \cdot x) d x,
$$

one obtains, using (47)

$$
\begin{aligned}
\hat{f}(\xi) & \approx \frac{1}{2 \pi} \int_{\Omega} f(x)\left[W_{J}\left(x, \rho_{\xi, J}\right)+W_{Y}\left(x, \rho_{\xi, Y}\right)\right] d x \\
& =\frac{1}{2 \pi} \int_{S}\left[\int_{\Omega} f(x) J_{0}(\lambda|y-x|) d x\right] \rho_{\xi, J}(y) d l(y) \\
& +\frac{1}{2 \pi} \int_{S}\left[\int_{\Omega} f(x) Y_{0}(\lambda|y-x|) d x\right] \rho_{\xi, Y}(y) d l(y),
\end{aligned}
$$


where the inner integrals are computed from the data $g$ :

$$
\begin{aligned}
\int_{\Omega} f(x) J_{0}(\lambda|y-x|) d x & =\int_{R^{+}} g(y, r) J_{0}(\lambda r) d r \\
\int_{\Omega} f(x) Y_{0}(\lambda|y-x|) d x & =\int_{R^{+}} g(y, r) Y_{0}(\lambda r) d r .
\end{aligned}
$$

Formula (48), in combination with (49) and (50), yields values of $\hat{f}(\xi)$ for arbitrary $\xi$. Now $f(x)$ can be recovered by numerically inverting the Fourier transform, or by a reduction to a FBP inversion $[73,88]$ of the regular Radon transform.

The most computationally expensive part of the algorithm, which is computing the densities $\rho_{\xi, J}$ and $\rho_{\xi, Y}$, needs to be done only once for a given acquisition surface. Thus, for a scanner with a fixed $S$, the resulting densities can be pre-computed once and for all. The actual reconstruction part then becomes extremely fast.

Examples of reconstructions from incomplete data using this technique of [83]) are shown in Figure 11. The images were reconstructed within the unit square $[-1,1] \times[-1,1]$, while the detectors were placed on the part of the concentric circle of radius 1.3 lying to the left of line $x_{1}=1$. We used the same phantom as in Figure 10(a)); the reconstruction from the data with added $15 \%$ noise is shown in Figure 11(b); part (c) demonstrates the results of applying additional smoothing filter to reduce the effects of noise in the data.

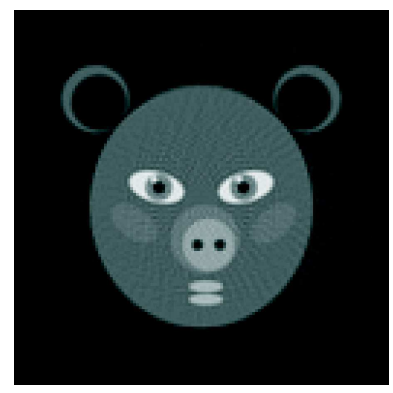

(a)

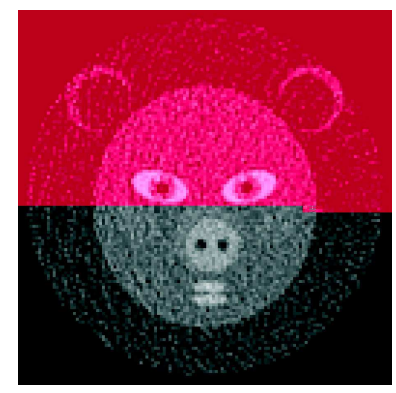

(b)

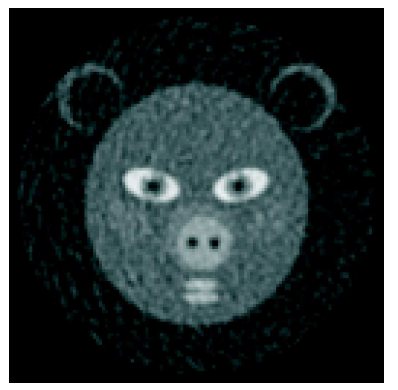

(c)

Figure 11: Examples of reconstruction from incomplete data using the technique of [83]. Detectors are located on the part of circular arc of radius 1.3 lying left of the line $x_{1}=1$. (a) reconstruction from accurate data (b) reconstruction from the data with added $15 \%$ noise (c) reconstruction from noisy data with additional smoothing filter

\subsubsection{Variable speed of sound}

The problem of numerical reconstruction in TAT from the data measured on open surfaces in the presence of a known variable speed of sound currently remains largely open. One of the difficulties was discussed in Section 3.3: even if the speed of sound $c(x)$ is nontrapping, it can happen that some of the characteristics escape from the region of interest to infinity without intersecting the open measuring surface. Then stable reconstruction of the corresponding interfaces will become impossible. It should be possible, however, to develop 
stable reconstruction algorithms in the case when the whole object of interest is located in the visible zone.

The generalization of the method of [83] to the case of variable speed of sound is so far problematic, since this algorithm is based on the knowledge of the open space Green's function for the Helmholtz equation. In the case of a non-constant $c(x)$, this Green's function is position-depended, and its numerical computation is likely to be prohibitively timeconsuming.

A promising approach to this problem, currently under development, is to use time reversal with the missing data replaced by zeros, or maybe by a more clever extension (e.g., using the range conditions, as in $[111,112]$ ). This would produce an initial approximation to $f(x)$, which one can try to refine by fixed-point iterations; however, the pertinent questions concerning such an algorithm remain open.

An interesting technique of using a reverberant cavity enclosing the target to compensate for the missing data is described in [31].

\section{$5 \quad$ Final remarks and open problems}

We list here some unresolved issues of mathematics of TAT/PAT, as well as some developments that were not addressed in the main text.

1. The issue of uniqueness acquisition sets $S$ (i.e., such that transducers distributed along $S$ provide sufficient information for TAT reconstruction) can be considered to be resolved, for most practical purposes. However, there remain significant unresolved theoretical questions. One of them consists of proving an analog of Theorem 8 for noncompactly supported functions with a sufficiently fast (e.g., super-exponential) decay at infinity. The original (and only known) proof of this theorem uses microlocal techniques $[7,122]$ that significantly rely upon the compactness of support. However, one hopes that the condition of a fast decay should suffice for this result. In particular, there is no proven analog of Theorem 6 for non-closed sets $S$ (unless $S$ is an open part of a closed analytic surface).

Techniques developed in [42] (see also [8] for their further use in TAT) might provide the right approach.

This also relates to still unresolved situation in dimensions 3 and higher. Namely, one would like to prove Conjecture 9 .

2. Concerning the inversion methods, one notices that closed form formulas are known only for spherical, cylindrical, and planar acquisition surfaces. The question arises whether closed form inversion formulas could be found for any other closed surface? It is the belief of the authors that the answer to this question is negative.

Another feature of the known closed form formulas that was mentioned before is that they do not work correctly if the support of the sought function $f(x)$ lies partially outside the acquisition surface. Time reversal and eigenfunction expansion methods do not suffer from this deficiency. The question arises whether one could find closed 
form formulas that reconstruct the function inside $S$ correctly, in spite of it having part of its support outside. Again, the authors believe that the answer is negative.

3. Besides algebraic iterative approaches, there are no reliable reconstruction methods in the case of the detectors partially surrounding the target, if the medium is acoustically inhomogeneous (see Section 4.2.2). This contrasts with the acoustically homogeneous situation (Section 4.2.1).

4. The complete range description of the forward operator $\mathcal{W}$ in even dimensions is still not known. It is also not clear whether one can obtain complete range descriptions for non-spherical observation sets $S$ or for a variable sound speed. The moment and orthogonality conditions do hold in the case of a constant speed and arbitrary closed surface, but they do not provide a complete description of the range. For acoustically inhomogeneous media, an analog of orthogonality conditions exists, but it also does not describe the range completely.

5. The problem of unique determination of the speed of sound from TAT data is largely open.

6. As it was explained in the text, knowing full Cauchy data of the pressure $p$ (i.e., its value and the value of its the normal derivative) on the observation surface $S$ leads to unique determination and simple reconstruction of $f$. However, the normal derivative is not measured by transducers and thus needs to be either found mathematically or measured in a different experiment. Thus, feasibility of techniques $[13,28]$ relying on full Cauchy data requires further mathematical and experimental study.

7. In the standard X-ray CT, as well as in SPECT, the so called local tomography technique $[37-39,77]$ is often very useful. It allows one to emphasize in a stable way singularities (e.g., tissue interfaces) of the reconstruction, even in the case of incomplete data (in the latter case, the invisible parts will be lost). An analog of local tomography can be easily implemented in TAT, for instance, by introducing an additional highfrequency filter in the FBP type formulas.

8. The mathematical analysis of TAT presented in the text did not take into account the issue of modeling and compensating for the acoustic attenuation. This subject is addressed in $[24,74,87,113,120]$, but probably cannot be considered completely resolved.

9. The initial pressure $f(x)$ that was the center of all discussions in the chapter (as well as in most papers devoted to TAT/PAT), is related, but is not exactly identical to the optical features of interest of the tissue. The issue of recovering the actual optical parameters of the tissue after the initial pressure $f(x)$ is found is non-trivial and is addressed, probably for the first time, in [20].

10. This chapter as well as most other papers devoted to TAT/PAT is centered on the initial pressure $f(x)$. This quantity is related, but is not exactly identical to the relevant optical features of the tissue. The problem of recovering the actual optical 
parameters of tissue (after $f(x)$ is found) is non-trivial and is addressed, probably for the first time, in [20].

11. The TAT technique discussed in the chapter uses active interrogation of the medium. There is a discussion in the literature of a passive version of TAT, where no irradiation of the target is involved [110].

\section{Acknowledgments}

The work of both authors was partially supported by the NSF DMS grant 0908208. The first author was also supported by the NSF DMS grant 0604778 and by the KAUST grant KUS-CI-016-04 through the IAMCS. The work of the second author was partially supported by the DOE grant DE-FG02-03ER25577. The authors express their gratitude to NSF, DOE, KAUST, and IAMCS for the support.

\section{References}

[1] M. Agranovsky, C. Berenstein, and P. Kuchment. Approximation by spherical waves in $L^{p}$-spaces. J. Geom. Anal. 6, (3):365-383, 1996.

[2] M. Agranovsky, D. Finch, and P. Kuchment, Range conditions for a spherical mean transform. Inverse Problems and Imaging 3(3) :373-38, 2009

[3] M. Agranovsky and P. Kuchment. Uniqueness of reconstruction and an inversion procedure for thermoacoustic and photoacoustic tomography with variable sound speed. Inverse Problems 23:2089-2102, 2007.

[4] M. Agranovsky, P. Kuchment, and L. Kunyansky. On reconstruction formulas and algorithms for the thermoacoustic and photoacoustic tomography, Ch. 8 in L. H. Wang (Editor) Photoacoustic imaging and spectroscopy, CRC Press 2009, pp. 89-101.

[5] M. Agranovsky, P. Kuchment, and E. T. Quinto. Range descriptions for the spherical mean Radon transform. J. Funct. Anal. 248: 344-386, 2007.

[6] M. Agranovsky and L. Nguyen. Range conditions for a spherical mean transform and global extension of solutions of Darboux equation. preprint arXiv:0904.4225 2009.

[7] M. Agranovsky and E. T. Quinto. Injectivity sets for the Radon transform over circles and complete systems of radial functions. J. Funct. Anal. 139:383-414, 1996.

[8] G. Ambartsoumian and P. Kuchment. On the injectivity of the circular Radon transform. Inverse Problems 21:473-485, 2005.

[9] G. Ambartsoumian and P. Kuchment. A range description for the planar circular Radon transform. SIAM J. Math. Anal. 38(2):681-692, 2006. 
[10] G. Ambartsoumian and S. Patch. Thermoacoustic tomography: numerical results. Proceedings of SPIE 6437, Photons Plus Ultrasound: Imaging and Sensing 200\%: The Eighth Conference on Biomedical Thermoacoustics, Optoacoustics, and Acousto-optics, Alexander A. Oraevsky, Lihong V. Wang, Editors, 64371B. 2007.

[11] H. Ammari, An Introduction to Mathematics of Emerging Biomedical Imaging, Springer Verlag, Berlin 2008.

[12] H. Ammari, E. Bonnetier, Y. Capdebosq, M. Tanter, and M. Fink. Electrical impedance tomography by elastic deformation. SIAM J. Appl. Math. 68(6):1557-1573, 2008 .

[13] H. Ammari, E. Bossy, V. Jugnon, and H. Kang, Mathematical Modelling in PhotoAcoustic Imaging SIAM Rev., to appear

[14] H. Ammari and H. Kang. Multi-Scale and Multi-Physics Biomedical Imaging Modalities, Chapter@@@ in this volume.

[15] M. A. Anastasio, J. Zhang, D. Modgil, and P. J. Rivière. Application of inverse source concepts to photoacoustic tomography Inverse Problems 23:S21-S35, 2007.

[16] M. A. Anastasio, J. Zhang, E. Y. Sidky, Z. Zou, X. Dan and X. Pan. Feasibility of Half-Data Image Reconstruction in 3-D Reflectivity Tomography With a Spherical Aperture, IEEE Transactions On Medical Imaging 24 (9): 1100-1112, 2005

[17] M. Anastasio, J. Zhang, X. Pan, Y. Zou, G. Ku and L. V. Wang. Half-time image reconstruction in thermoacoustic tomography. IEEE Trans. Med. Imaging 24:199-210, 2005 .

[18] L.-E.Andersson. On the determination of a function from spherical averages. SIAM J. Math. Anal. 19(1):214-232, 1988.

[19] V. Andreev, D. Popov, et al. Image reconstruction in 3D optoacoustic tomography system with hemispherical transducer array. Proc. SPIE 4618:137:145, 2002.

[20] G. Bal, A. Jollivet, and V. Jugnon. Inverse Transport Theory of Photoacoustics. preprint, arXiv:0908.4012, 2009.

[21] A. G. Bell. On the production and reproduction of sound by light. Am. J. Sci. 20:305$324,1880$.

[22] G. Beylkin. The inversion problem and applications of the generalized Radon transform. Comm. Pure Appl. Math. 37:579-599, 1984.

[23] T. Bowen. Radiation-induced thermoacoustic soft tissue imaging. Proc. IEEE Ultrasonics Symposium 2:817-822, 1981.

[24] P. Burgholzer, H. Grün, M. Haltmeier, R. Nuster, and G. Paltauf. Compensation of acoustic attenuation for high-resolution photoacoustic imaging with line detectors using time reversal. Proc. SPIE number 6437-75 Photonics West, BIOS 2007, San Jose/California, USA. 2007. 
[25] P. Burgholzer, C. Hofer, G. Paltauf, M. Haltmeier, O. Scherzer. Thermoacoustic tomography with integrating area and line detectors. IEEE Transactions on Ultrasonics, Ferroelectrics, and Frequency Control 52(9):1577-1583, 2005.

[26] P. Burgholzer, C. Hofer, G. J. Matt, G. Paltauf, M. Haltmeier, and O. Scherzer. Thermoacoustic tomography using a fiber-based Fabry-Perot interferometer as an integrating line detector. Proc. SPIE 6086, 434-442. 2006

[27] M. Cheney. A Mathematical Tutorial on Synthetic Aperture Radar. SIAM Review. 43(2): 301-312, 2001.

[28] C. Clason and M. Klibanov. The quasi-reversibility method in thermoacoustic tomography in a heterogeneous medium. SIAM J. Scientific Computing. 30: 1-23, 2007

[29] D. Colton, L. Paivarinta, and J. Sylvester. The interior transmission problem Inverse Problems 1(1): 13-28, 2007.

[30] Courant, R. and Hilbert, D. Methods of Mathematical Physics, Volume II Partial Differential Equations. Interscience, New York, 1962.

[31] Cox, B. T., Arridge, S. R. and Beard, P. C. Photoacoustic tomography with a limitedaperture planar sensor and a reverberant cavity. Inverse Problems 23: S95-S112, 2007

[32] A. J. Devaney and G. Beylkin. Diffraction tomography using arbitrary transmitter and receiver surfaces. Ultrasonic Imaging 6: 181-193, 1984.

[33] G. J. Diebold, T. Sun, and M. I. Khan. Photoacoustic monopole radiation in one, two, and three dimensions. Phys. Rev. Lett. 67(24): 3384-3387, 1991.

[34] Yu. V. Egorov and M. A. Shubin. Partial Differential Equations I. Encyclopaedia of Mathematical Sciences, (Springer Verlag), 30:1-259, 1992.

[35] Ehrenpreis, L. The Universality of the Radon Transform, Oxford Univ. Press, 2003.

[36] L. Evans, Partial Differential Equations, Amer. Math. Soc., Providence, 1988.

[37] A. Faridani, E. L. Ritman, and K. T. Smith. Local tomography. SIAM J. Appl. Math 52(4): 459-484, 1992.

[38] A. Faridani, E. L. Ritman, and K. T. Smith. Examples of local tomography. SIAM J. Appl. Math 52(4): 1193-1198, 1992.

[39] A. Faridani, D.V. Finch, E.L. Ritman, and K. Smith, Local Tomography II, SIAM J. Appl. Math. 57: 1095-1127, 1997.

[40] J. A. Fawcett. Inversion of $n$-dimensional spherical averages. SIAM J. Appl. Math. 45(2): 336-341, 1985.

[41] Finch, D., Haltmeier, M. and Rakesh. Inversion of spherical means and the wave equation in even dimensions. SIAM J. Appl. Math. 68(2): 392-412, 2007. 
[42] D. Finch, S. Patch and Rakesh. Determining a function from its mean values over a family of spheres. SIAM J. Math. Anal. 35(5): 1213-1240, 2004.

[43] D. Finch and Rakesh. Range of the spherical mean value operator for functions supported in a ball. Inverse Problems 22: 923-938, 2006.

[44] D. Finch and Rakesh. Recovering a function from its spherical mean values in two and three dimensions. In [133], 77-88.

[45] D. Finch and Rakesh. The spherical mean value operator with centers on a sphere. Inverse Problems 23(6): S37-S50, 2007.

[46] B. Gebauer and O. Scherzer, Impedance-Acoustic Tomography. SIAM J. Applied Math. 69(2): 565-576, 2009.

[47] I. Gelfand, S. Gindikin and M. Graev M. Integral geometry in affine and projective spaces. J. Sov. Math. 18: 39-167, 1980.

[48] I. Gelfand, S. Gindikin and M. Graev M. Selected Topics in Integral Geometry. Transl. Math. Monogr. v. 220, Amer. Math. Soc., Providence RI, 2003.

[49] I. Gelfand, M. Graev M. and N. Vilenknin. Generalized Functions, v. 5: Integral Geometry and Representation Theory, Acad. Press. 1965.

[50] S. Gindikin. Integral geometry on real quadrics, in Lie groups and Lie algebras: E. B. Dynkin's Seminar, 23-31, Amer. Math. Soc. Transl. Ser. 2, 169, Amer. Math. Soc., Providence, RI, 1995.

[51] A. Greenleaf and G. Uhlmann. Microlocal techniques in integral geometry. Contemporary Math. 113: 149-155, 1990.

[52] H. Grün, M. Haltmeier, G. Paltauf and P. Burgholzer. Photoacoustic tomography using a fiber based Fabry-Perot interferometer as an integrating line detector and image reconstruction by model-based time reversal method. Proc. SPIE 6631:663107, 2007.

[53] V. Guillemin. Fourier integral operators from the Radon transform point of view. Proc. Symposia in Pure Math. 27: 297-300, 1975.

[54] V. Guillemin. On some results of Gelfand in integral geometry. Proc. Symposia in Pure Math. 43: 149-155, 1985.

[55] V. Guillemin and S. Sternberg. Geometric Asymptotics . Amer. Math. Soc., Providence, RI, 1977.

[56] M. Haltmeier, P. Burgholzer, G. Paltauf, and O. Scherzer. Thermoacoustic computed tomography with large planar receivers. Inverse Problems 20: 1663-1673, 2004.

[57] M. Haltmeier, T. Schuster and O. Scherzer. Filtered backprojection for thermoacoustic computed tomography in spherical geometry. Mathematical Methods in the Applied Sciences 28: 1919-1937, 2005. 
[58] M. Haltmeier, G. Paltauf, P. Burgholzer, and O. Scherzer. Thermoacoustic Tomography with integrating line detectors. Proc. SPIE 5864:586402-8, 2005.

[59] M. Haltmeier, P. Burgholzer, C. Hofer, G. Paltauf, R. Nuster, and O. Scherzer. Thermoacoustic tomography using integrating line detectors. Ultrasonics Symposium 1:166$169,2005$.

[60] M. Haltmeier, O. Scherzer, P. Burgholzer, and G. Paltauf. Thermoacoustic Computed Tomography with large planar receivers. ECMI Newsletter 37: 31-34, 2005. http://www.it.lut.fi/mat/EcmiNL/ecmi37/

[61] M. Haltmeier and T. Fidler. Mathematical Challenges Arising in Thermoacoustic Tomography with Line Detectors, preprint arXiv:math.AP/0610155.

[62] M. Haltmeier, O. Scherzer, P. Burgholzer, R. Nuster, and G. Paltauf. Thermoacoustic Tomography And The Circular Radon Transform: Exact Inversion Formula. Mathematical Models and Methods in Applied Sciences 17(4): 635655, 2007.

[63] s. Helgason. The Radon Transform, Birkh äuser, Basel, 1980.

[64] s. Helgason. Groups and Geometric Analysis . Amer. Math. Soc., Providence, R.I, 2000 .

[65] Herman, G.(Ed.) Image Reconstruction from Projections . Topics in Applied Physics, v. 32, Springer Verlag, Berlin, New York, 1979.

[66] 1. Hörmander. The Analysis of Linear Partial Differential Operators, vol. 1, 2, SpringerVerlag, New York, 1983.

[67] Y. Hristova. Time reversal in thermoacoustic tomographyan error estimate. Inverse Problems 25: 1-14, 2009.

[68] Y. Hristova, P. Kuchment, and L. Nguyen. On reconstruction and time reversal in thermoacoustic tomography in homogeneous and non-homogeneous acoustic media, Inverse Problems 24: 055006, 2008.

[69] Inverse Problems, a special issue devoted to thermoacoustic tomography, 23(6), 2007.

[70] V. Isakov. Inverse Problems for Partial Differential Equations, 2nd edition, Springer verlag, Berlin 2005.

[71] X. Jin and L. V Wang. Thermoacoustic tomography with correction for acoustic speed variations. Physics in Medicine and Biology 51:6437-6448, 2006.

[72] F. John. Plane Waves and Spherical Means Applied to Partial Differential Equations, Dover, 1971.

[73] A. C. Kak and M. Slaney. Principles of Computerized Tomographic Imaging. SIAM, Philadelphia, 2001. 
[74] R. Kowar, O. Scherzer, and X. Bonnefond. Causality Analysis of Frequency Dependent Wave Attenuation, preprint arXiv:0906.4678.

[75] R. A. Kruger, P. Liu, Y. R. Fang, and C. R. Appledorn. Photoacoustic ultrasound (PAUS)reconstruction tomography. Med. Phys. 22: 1605-1609, 1995.

[76] P. Kuchment. Generalized Transforms of Radon Type and Their Applications. in [96], 67-91, 2006.

[77] Kuchment, P., Lancaster, K. and Mogilevskaya, L. On local tomography. Inverse Problems, 11: 571-589, 1995.

[78] P. Kuchment and L. Kunyansky. Mathematics of thermoacoustic tomography, European J. Appl. Math. 19(02): 191-224, 2008.

[79] P. Kuchment and L. Kunyansky. Synthetic focusing in ultrasound modulated tomography, to appear in Inverse Problems and Imaging.

[80] P. Kuchment and E. T. Quinto. Some problems of integral geometry arising in tomography. Chapter XI in [35], 2003.

[81] L. Kunyansky. Explicit inversion formulae for the spherical mean Radon transform. Inverse problems. 23: 737-783, 2007.

[82] L. Kunyansky. A series solution and a fast algorithm for the inversion of the spherical mean Radon transform. Inverse Problems. 23: S11-S20, 2007.

[83] L. Kunyansky, Thermoacoustic tomography with detectors on an open curve: an efficient reconstruction algorithm. Inverse Problems 24(5):055021, 2008.

[84] V. Lin and A. Pinkus. Fundamentality of ridge functions. J. Approx. Theory. 75: 295311, 1993.

[85] V. Lin and A. Pinkus. Approximation of multivariate functions. In Advances in Computational Mathematics, H. P. Dikshit and C. A. Micchelli, Eds., World Sci. Publ., 1-9, 1994.

[86] A. K. Louis and E. T. Quinto. Local tomographic methods in Sonar. In Surveys on solution methods for inverse problems, Springer, Vienna, 147-154, 2000.

[87] K. Maslov, H. F. Zhang and L. V. Wang. Effects of wavelength-dependent fluence attenuation on the noninvasive photoacoustic imaging of hemoglobin oxygen saturation in subcutaneous vasculature in vivo. Inverse Problems 23: S113-S122, 2007.

[88] F. Natterer. The mathematics of computerized tomography, Wiley, New York, 1986.

[89] F. Natterer and F. Wübbeling. Mathematical Methods in Image Reconstruction, Monographs on Mathematical Modeling and Computation 5, SIAM, Philadelphia, PA, 2001.

[90] L. Nguyen, A family of inversion formulas in thermoacoustic tomography. Inverse Problems and Imaging 3(4): 649-675, 2009. 
[91] L. V. Nguyen. On singularities and instability of reconstruction in thermoacoustic tomography, preprint arXiv:0911.5521v1.

[92] Nilsson, S. Application of fast backprojection techniques for some inverse problems of integral geometry. Linkoeping studies in science and technology, Dissertation 499, Dept. of Mathematics, Linkoeping university, Linkoeping, Sweden. 1997.

[93] C. J. Nolan and M. Cheney, M. Synthetic aperture inversion. Inverse Problems 18: 221-235, 2002.

[94] S. J. Norton. Reconstruction of a two-dimensional reflecting medium over a circular domain: exact solution. J. Acoust. Soc. Am. 67: 1266-1273, 1980.

[95] S. J. Norton and M. Linzer. Ultrasonic reflectivity imaging in three dimensions: exact inverse scattering solutions for plane, cylindrical, and spherical apertures. IEEE Transactions on Biomedical Engineering. 28: 200-202, 1981.

[96] Olafsson, G. and Quinto, E. T. (Editors), The Radon Transform, Inverse Problems, and Tomography. American Mathematical Society Short Course January 3-4, 2005, Atlanta, Georgia, Proc. Symp. Appl. Math. 63, AMS, RI, 2006.

[97] A. A. Oraevsky, R. O. Esenaliev, S. L. Jacques, and F. K. Tittel. Laser optoacoustic tomography for medical diagnostics principles. Proc. SPIE 2676, 22. 1996.

[98] A. A. Oraevsky, S. L. Jacques, R. O. Esenaliev, and F. K. Tittel, Laser-based ptoacoustic imaging in biological tissues, Proc. SPIE 2134A:122-128, 1994.

[99] A. A. Oraevsky and A. A. Karabutov. In Handbook of Optical Biomedical Diagonstics, edited by V. V. Tuchin, SPIE, Bellingham, WA, Chap. 10, 2002.

[100] A. A. Oraevsky and A. A. Karabutov. Optoacoustic Tomography, Ch. 34 In Biomedical Photonics Handbook, edited by T. Vo-Dinh, CRC, Boca Raton, FL, Chap. 34, 34-1 34-34, 2003.

[101] V. P. Palamodov. Reconstruction from limited data of arc means. J. Fourier Anal. Appl. 6(1): 25-42, 2000.

[102] V. P. Palamodov. Reconstructive Integral Geometry. Birkhäuser, Basel. 2004.

[103] V. Palamodov. Remarks on the general Funk-Radon transform and thermoacoustic tomography. Preprint arxiv: math.AP/0701204, 2007.

[104] G. Paltauf, P. Burgholzer, M. Haltmeier and O. Scherzer. Thermoacoustic Tomography using optical Line detection. Proc. SPIE 5864: 7-14, 2005.

[105] G. Paltauf, R. Nuster, and P. Burgholzer Weight factors for limited angle photoacoustic tomography, Phys. Med. Biol. 54: 3303-3314, 2009.

[106] G. Paltauf, R. Nuster, M. Haltmeier, and P. Burgholzer. Thermoacoustic Computed Tomography using a Mach-Zehnder interferometer as acoustic line detector. Appl. Opt. 46(16): 3352-8, 2007. 
[107] G. Paltauf, R. Nuster, M. Haltmeier and P. Burgholzer. Experimental evaluation of reconstruction algorithms for limited view photoacoustic tomography with line detectors. Inverse Problems 23: S81S94, 2007.

[108] G. Paltauf, J. A. Viator, S. A. Prahl, and S. L. Jacques. Iterative reconstruction algorithm for optoacoustic imaging J. Acoust. Soc. Am. 112(4): 1536-1544, 2002.

[109] G. Paltauf, R. Nuster, and P. Burgholzer. Characterization of integrating ultrasound detectors for photoacoustic tomography Journal Of Applied Physics. 105:102026, 2009.

[110] V. I. Passechnik, A. A. Anosov and K. M. Bograchev. Fundamentals and prospects of passive thermoacoustic tomography. Critical reviews in Biomed. Eng. 28(3-4): 603-640, 2000 .

[111] S. K. Patch. Thermoacoustic tomography - consistency conditions and the partial scan problem. Phys. Med. Biol. 49:1-11, 2004.

[112] S. Patch. Photoacoustic or thermoacoustic tomography: consistency conditions and the partial scan problem, in [133], 103-116, 2009.

[113] S. K. Patch and M. Haltmeier. Thermoacoustic Tomography - Ultrasound Attenuation Artifacts IEEE Nuclear Science Symposium Conference 4: 2604-2606, 2006.

[114] S. K. Patch and O. Scherzer. Photo- and Thermo-Acoustic Imaging (Guest Editors' Introduction). Inverse Problems 23:S01-S10, 2007.

[115] D. A. Popov and D. V. Sushko. A parametrix for the problem of optical-acoustic tomography. Dokl. Math. 65(1): 19-21, 2002.

[116] D. A. Popov and D. V. Sushko. Image restoration in optical-acoustic tomography. Problems of Information Transmission 40(3): 254-278, 2004.

[117] E. T. Quinto. The dependence of the generalized Radon transform on defining measures. Trans. Amer. Math. Soc. 257:331-346, 1980.

[118] E. T. Quinto. Singularities of the X-ray transform and limited data tomography in $\mathbb{R}^{2}$ and $\mathbb{R}^{3}$. SIAM J. Math. Anal. 24: 1215-1225, 1993.

[119] E. T. Quinto.An introduction to X-ray tomography and Radon transforms. In [96], 1-23, 2006.

[120] P. J. La Rivière, J. Zhang and M. A. Anastasio. Image reconstruction in optoacoustic tomography for dispersive acoustic media. Optics Letters 31(6): 781-783, 2006.

[121] M. A. Shubin, Pseudodifferential Operators and Spectral Theory, Springer Verlag, Berlin 2001.

[122] P. Stefanov and G. Uhlmann. Integral geometry of tensor fields on a class of non-simple Riemannian manifolds. Amer. J. Math. 130(1): 239-268, 2008. 
[123] P. Stefanov and G. Uhlmann. Thermoacoustic tomography with variable sound speed. Inverse Problems 25:075011, 2009.

[124] D. Steinhauer. A uniqueness theorem for thermoacoustic tomography in the case of limited boundary data, preprint arXiv:0902.2838.

[125] D. Steinhauer. A reconstruction procedure for thermoacoustic tomography in the case of limited boundary data, preprint arXiv:0905.2954.

[126] R. S. Strichartz. A Guide to Distribution Theory and Fourier Transforms. World. Sci., 2003.

[127] A. C. Tam. Applications of photoacoustic sensing techniques. Rev. Mod. Phys. 58(2): 381-431, 1986.

[128] Tuchin, V. V. (Editor) Handbook of Optical Biomedical Diagnostics. SPIE, Bellingham, WA, 2002.

[129] B. Vainberg. The short-wave asymptotic behavior of the solutions of stationary problems, and the asymptotic behavior as $t \rightarrow \infty$ of the solutions of nonstationary problems. Russian Math. Surveys. 30(2): 1-58, 1975.

[130] B. Vainberg Asymptotics methods in the Equations of Mathematical Physics. Gordon and Breach, 1982.

[131] T. Vo-Dinh, (Editor). Biomedical Photonics Handbook. CRC, Boca Raton, FL, 2003.

[132] Kun, Wang, and M. A. Anastasio. Photoacoustic and Thermoacoustic Tomography: Image Formation Principles, Chapter @@@ in this volume.

[133] L. Wang, (Editor) Photoacoustic imaging and spectroscopy, CRC Press, Boca Raton, FL, 2009.

[134] Wang, L. V. Wang and H. Wu. Biomedical Optics. Principles and Imaging. WileyInterscience, 2007.

[135] M. Xu and L.-H. V. Wang. Time-domain reconstruction for thermoacoustic tomography in a spherical geometry. IEEE Trans. Med. Imag. 21: 814-822, 2002.

[136] M. Xu and L.-H. V. Wang. Universal back-projection algorithm for photoacoustic computed tomography. Phys. Rev. E 71:016706, 2005.

[137] M. Xu and L.-H. V. Wang. Photoacoustic imaging in biomedicine. Review of Scientific Instruments 77:041101-01 - 041101-22, 2006.

[138] Y. Xu, D. Feng and L.-H. V. Wang. Exact frequency-domain reconstruction for thermoacoustic tomography: I. Planar geometry. IEEE Trans. Med. Imag. 21: 823-828, 2002. 
[139] Y. Xu, M. Xu and L.-H. V. Wang. Exact frequency-domain reconstruction for thermoacoustic tomography: II. Cylindrical geometry. IEEE Trans. Med. Imag. 21: 829-833, 2002.

[140] Y. Xu, L., Wang, G. Ambartsoumian, and P. Kuchment. Reconstructions in limited view thermoacoustic tomography. Medical Physics. 31(4): 724-733, 2004.

[141] Y. Xu, L. Wang, G. Ambartsoumian, and P. Kuchment. Limited view thermoacoustic tomography, Ch. 6 in L. H. Wang (Editor) Photoacoustic imaging and spectroscopy, CRC Press 2009, pp. 61-73.

[142] G. Zangerl, O. Scherzer and M. Haltmeier. Circular integrating detectors in photo and thermoacoustic tomography, Inverse Problems in Science and Engineering. 17(1): 133142, 2009.

[143] Z. Yuan, Q. Zhang, and H. Jiang. Simultaneous reconstruction of acoustic and optical properties of heterogeneous media by quantitative photoacoustic tomography. Optics Express 14(15): 6749, 2006

[144] J. Zhang and M. A. Anastasio. Reconstruction of speed-of-sound and electromagnetic absorption distributions in photoacoustic tomography. Proc. SPIE 6086: 608619, 2006. 